\title{
Neutralizing breadth of antibodies targeting diverse conserved epitopes between SARS-CoV and SARS- CoV-2
}

\section{Hua-Long Xiong}

National Institute of Diagnostics and Vaccine Development in Infectious Diseases, School of Public Health \& School of Life Science, Xiamen University

Hui Sun

Xiamen University

Si-Ling Wang

Xiamen University

\section{Lunzhi Yuan}

School of Public Health, Xiamen University, Xiamen, PR China.

\section{Liqin Liu}

Xiamen University

\section{Yuhe Zhu}

Xiamen University

Jinlei Zhang

Xiamen University

Yang Huang

Xiamen University

Ruoyao Qi

Xiamen University

Yao Jiang

Xiamen University

Jian Ma

Xiamen University

\section{Min Zhou}

Xiamen University

\section{Yue Ma}

Xiamen University

\section{Rao Fu}

Xiamen University

\section{Siping Yan}

Xiamen University 


\section{Mingxi Yue}

State Key Laboratory of Molecular Vaccinology and Molecular Diagnostics, School of Life Sciences,

Xiamen University

\section{Yangtao Wu}

State Key Laboratory of Molecular Vaccinology and Molecular Diagnostics, National Institute of

Diagnostics and Vaccine Development in Infectious Diseases, School of Public Health, Xiamen University

\section{Min Wei}

Xiamen University

\section{Yi-Zhen Wang}

Xiamen University

\section{Tingting Li}

Xiamen University https://orcid.org/0000-0002-9917-7804

\section{Yingbin Wang}

State Key Laboratory of Molecular Vaccinology and Molecular Diagnostics, School of Life Sciences, School of Public Health, Xiamen University, Xiamen, 361102

\section{Zi-Zheng Zheng}

Xiamen University https://orcid.org/0000-0003-0099-4212

\section{Hai Yu}

State Key Laboratory of Molecular Vaccinology and Molecular Diagnostics, School of Life Sciences, Xiamen University, Xiamen

\section{Tong Cheng}

Xiamen University https://orcid.org/0000-0002-1638-6214

\section{Shaowei Li}

Xiamen University https://orcid.org/0000-0002-3374-1038

\section{Quan Yuan}

Xiamen University https://orcid.org/0000-0001-5487-561X

\section{Jun Zhang}

Xiamen University https://orcid.org/0000-0002-6601-9180

\section{Yi Guan}

Shantou University Medical College

\section{Qingbing Zheng}

Xiamen University https://orcid.org/0000-0002-7516-9965

\section{Tianying Zhang}

Xiamen University

\section{Ningshao Xia ( $\square$ nsxia@xmu.edu.cn )}

Xiamen University https://orcid.org/0000-0003-0179-5266

\section{Article}


Keywords:

Posted Date: February 24th, 2022

DOI: https://doi.org/10.21203/rs.3.rs-1386044/v1

License: (c) (1) This work is licensed under a Creative Commons Attribution 4.0 International License. Read Full License 
Neutralizing breadth of antibodies targeting diverse conserved epitopes between SARS-CoV and SARS-CoV-2

Hualong Xiong ${ }^{1, \#, ~ H u i ~ S u n ~}{ }^{1, \#}$, Siling Wang ${ }^{1, \#, ~ L u n z h i ~ Y u a n ~}{ }^{1, \#}$, Liqin Liü, \#, Yuhe Zhu1, \#, Jinlei Zhang ${ }^{1}$, , Yang Huang ${ }^{1}$, Ruoyao Qi ${ }^{1}$, Yao Jiang ${ }^{1}$, Jian Ma ${ }^{1}$, Ming Zhou ${ }^{1}$, Yue $\mathrm{Ma}^{1}$, Rao $\mathrm{Fu}^{1}$, Siping Yan ${ }^{1}$, Mingxi Yue ${ }^{1}$, Yangtao $\mathrm{Wu}^{1}$, Min $\mathrm{Wei}^{1}$, Yizhen Wang ${ }^{1}$, Tingting $\mathrm{Li}^{1}$, Yingbin Wang ${ }^{1}$, Zizheng Zheng ${ }^{1}$, Hai $\mathrm{Yu}^{1}$, Tong Cheng ${ }^{1}$, Shaowei $\mathrm{Li}^{1}{ }^{\text {* }}$,

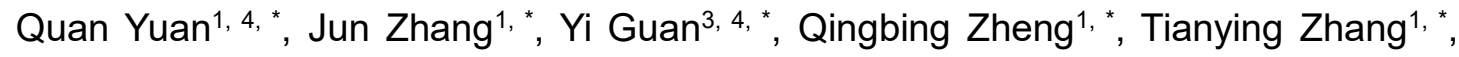
Ningshao $\mathrm{Xia}^{1,2, *}$

${ }^{1}$ State Key Laboratory of Molecular Vaccinology and Molecular Diagnostics; National Institute of Diagnostics and Vaccine Development in Infectious Diseases, School of Public Health, School of Life Sciences, Xiamen University, Xiamen 361102, China ${ }^{2}$ Research Unit of Frontier Technology of Structural Vaccinology, Chinese Academy of Medical Sciences, Xiamen 361102, China

${ }^{3}$ State Key Laboratory of Emerging Infectious Diseases, University of Hong Kong, Hong Kong 999077, China.

4 Guangdong-Hongkong Joint Laboratory of Emerging Infectious Diseases/Joint Laboratory for International Collaboration in Virology and Emerging Infectious Diseases, Joint Institute of Virology (Shantou University and University of Hong Kong), Shantou University, Shantou 515063, China.

* Corresponding authors: shaowei@xmu.edu.cn (S.L.), yuanquan@xmu.edu.cn (Q.Y.), zhangi@xmu.edu.cn (J.Z.), yguan@hku.hk (Y.G.), abing0811@xmu.edu.cn (Q.Z.), zhangtianying@xmu.edu.cn (T.Z.), nsxia@xmu.edu.cn (N.X.)

\# These authors contributed equally. 


\section{Abstract:}

Antibody therapeutics for the treatment of COVID-19 has been highly successful while faces a challenge of the recent emergence of the Omicron variant which escapes the majority of existing SARS-CoV-2 neutralizing antibodies (nAbs). Here, we successfully generated a panel of SARS-CoV-2/SARS-CoV cross-neutralizing antibodies by sequential immunization of the two pseudoviruses. Of which, nAbs X01, X10 and X17 showed broadly neutralizing breadths against most variants of concern (VOCs) and $\mathrm{X} 17$ was further identified as a Class $5 \mathrm{nAb}$ with undiminished neutralization against the Omicron variant. Cryo-EM structures of three-antibody in complex with the spike proteins of prototyped SARS-CoV-2, Delta, Omicron and SARS-CoV defined three non-overlapping conserved epitopes on the receptor-binding domain (RBD). The triple antibody cocktail exhibited enhanced resistance to viral escape and effective protection against the infection of Beta variant in hamsters. Our finding will aid the development of both antibody therapeutics and broad vaccines against SARS-CoV-2 and emerging variants. 


\section{Introduction}

As of January 2022, the coronavirus disease 2019 (COVID-19) pandemic, caused by the severe acute respiratory syndrome coronavirus 2 (SARS-CoV-2) has resulted in over 5 million deaths worldwide ${ }^{1-3}$. Monoclonal antibodies (mAbs) isolated from SARSCoV-2 infected individuals have exhibited effectiveness as both therapeutics or prophylactics against SARS-CoV-2 ${ }^{4-6}$, thus many neutralizing antibodies (nAbs), e.g. sotrovimab ${ }^{7}$ and bamlanivimab ${ }^{8}$ and nAb cocktails, e.g. casirivimab-imdevimab ${ }^{9}$ and bamlanivimab-etesevimab ${ }^{10}$ have been in Emergency Use Authorization (EUA) for treatment of COVID-19 in patients. However, constant evolution and genetic drift of SARS-CoV-2 has resulted in the emergence of many variants of concern (VOCs), including Alpha (B.1.1.7), Beta (B.1.351), Gamma (B.1.1.28), Delta (B.1.617.2) and Omicron (B.1.1.529) variants, the latter become the major concern of multiple countermeasures depending on the main protein of SARS-CoV-2 prototype strain. Shockingly, the Omicron variant accumulates a lot of residue substitutions in the spike (S) protein, of which 15 mutations are highly intertwined with common neutralizing epitopes in the receptor-binding domain $(R B D)^{11,12}$. As reported, some critical mutations of SARS-CoV-2 VOCs could destroy the neutralization of mAbs potently neutralizing ancestral isolate, revealing that the protective efficacy of antibody therapeutics might be diminished ${ }^{13-20}$. Therefore, it is urgently required for $\mathrm{nAbs}$ with broader neutralizing breadth against current VOCs and future emerging variants.

The trimeric S protein mediates SARS-CoV-2 entry into host cells by the RBD that binds to the angiotensin-converting enzyme 2 (ACE2) receptor ${ }^{1,21,22}$. Since RBD is a 
critical trigger factor inducing SARS-CoV-2 infection, thus has been identified as the main target for therapeutics and vaccine development against COVID-19. A large number of potently neutralizing mAbs have been identified which mainly target the receptor-binding motif ( $R B M)$ on the $R B D$ to efficiently inhibit $S$ protein binding to ACE2 ${ }^{4,23,24}$. However, mutant residues of VOCs usually reside in the RBM, significantly reducing the neutralization breadth of mAbs recognizing this site ${ }^{13-18,25}$. Nevertheless, of all five identified classes of RBD-targeting $n A b s^{4,26}$, three classes, represented by $\mathrm{S} 309^{27}, \mathrm{~S} 2 \mathrm{X} 259^{28}$ and $\mathrm{S} 2 \mathrm{H} 97^{29}$, have been shown to cross neutralize SARS-CoV-2 and SARS-CoV, and further inhibit infection of most VOCs, revealing that epitopes within these sites are highly conserved among Sarbecoviruses. Antibody cocktail composed of representative nAbs binding to these conserved epitopes possibly have the potential to prevent SARS-CoV-2 variants and spillover SARS-like virus. Additionally, under pressure screening of antibody therapeutics, the emergence of escape mutation becomes an important issue to be considered. Escape studies in vitro have strongly supported the rationale of antibody cocktails consisting of noncompeting antibodies to avoid resistance ${ }^{13,15,30}$.

Previously reported nAbs are mainly obtained from human humoral immune response induced by vaccination or natural infection of SARS-CoV or SARS-CoV-2. The temporary exposure of single Sarbecoviruses hinders the generation of crossneutralizing $m \mathrm{mAbs}^{27-29}$. Based on the experience of influenza virus research ${ }^{31-33}$, combination immunization of SARS-CoV and SARS-CoV-2 in sequence may play an important role in the immune-focusing on the conserved epitopes to develop cross- 
neutralizing antibodies.

In this study, we focus on the conserved epitopes between SARS-CoV-2 and SARS-CoV, generate a panel of broad neutralizing antibodies (bnAbs) against SARSCoV, SARS-CoV-2 and VOCs from sequential immunized mice. Three representative bnAbs, X01, X10 and X17, are further identified potently cross-neutralize most VOCs but with decreased neutralization against Omicron in vary degree. High-resolution cryo-electron microscopy (cryo-EM) structures reveal three non-overlapped conserved epitopes and define the structural basis for the neutralization breadths of three bnAbs. Furthermore, the triple antibody efficiently resists to viral escape and protects Syrian hamsters against SARS-CoV-2 Beta variant challenge. Thus, our results expand the therapeutics strategy, based on conserved epitopes, to cope with circulating and future emerging SARS-CoV-2 VOCs and even spillover of SARS-like virus, and highlight the potential of diverse conserved epitopes in vaccine design.

\section{Results}

Sequential immunization of SARS-CoV and SARS-CoV-2 elicits crossneutralizing antibodies

To efficiently generate SARS-CoV-2 and SARS-CoV cross-neutralizing mAbs targeting RBD, we implemented SARS-CoV and SARS-CoV-2 S proteins which were carried on recombinant vesicular stomatitis virus (VSV) pseudovirus, termed as rVSV-SARS and rVSV-SARS2, respectively, as previously reported $^{34}$. The mice were alternately immunized with these two purified pseudoviruses at a one-week interval, of which 
rVSV-SARS acted as the priming immunogen (Fig. S1). After three doses of both rVSV-SARS and rVSV-SARS2, the sequential immunized mice were sacrificed to build the hybridoma cell pools for the selection of cross-neutralizing mAbs against SARSCoV and SARS-CoV-2. Finally, a total of 34 cross-neutralizing mAbs were obtained for further evaluation.

According to the diversity of the neutralization against SARS-CoV and SARSCoV-2, 34 cross-neutralizing mAbs were further classified into 3 classes, termed as C1, C2 and C3 (Fig. 1A and Table S1). A majority of mAbs (19 of 34) were classified into $\mathrm{C} 1$, which showed comparable neutralizing efficacies against SARS-CoV-2 and SARS-CoV with half-maximal inhibitory concentration $\left(\mathrm{IC}_{50}\right)$ values differed within one order of magnitude, revealing that these cross-neutralizing mAbs recognize epitopes highly conserved within SARS-CoV-2 and SARS-CoV. Conversely, C2 and C3 contain weak cross-neutralizing mAbs with biased neutralization potencies against SARS-CoV and SARS-CoV-2, respectively (Fig. 1B, C and Table S1). We next determined the broadly neutralizing potencies of those mAbs against pseudoviruses of VOCs including B.1.1.7 (Alpha), B.1.351 (Beta), B.1.1.28 (Gamma), and B.1.617.2 (Delta). Of note, the cross-neutralizing mAbs in $\mathrm{C} 1$ showed comparable neutralizing efficacies against pseudoviruses of VOCs when compared to D614G (Fig. 1B, left panel), with $\mathrm{IC}_{50}$ values differing within one order of magnitude (Fig. 1C, left panel). These results indicated that nAbs in $\mathrm{C} 1$ not only ensure strong neutralization activities, but also effectively avoid the escape of SARS-CoV-2 variants. In contrast, mAbs in C2 showed limited neutralizing potencies to SARS-CoV-2 and VOCs (Fig. 1B and C, middle 
panels), and SARS-CoV-2 biased nAbs in $\mathrm{C} 3$, although with better neutralizing efficacies against SARS-CoV-2 (Fig. 1B, right panel), showed less resist to VOCs (Fig. 1C, right panel), comparing to those nAbs in $\mathrm{C} 1$.

According to the above information, it is inferred that nAbs in $\mathrm{C} 1$ might be resistant to a variety of mutations found in VOCs as well as VOls. To this end, we sought to investigate the single mutations on RBD for their influence on mAbs neutralization. $A$ total of 55 RBD single-point mutations with high frequency since the COVID-19 outbreak were included in this study. Then, a set of corresponding 55 mutant SARSCoV-2 pseudoviruses carrying these single-point mutations were constructed to evaluate all three classes of nAbs. Neutralization results were similar to that assessed against VOCs. Of these 3 class nAbs, $\mathrm{C} 1 \mathrm{nAbs}$ resistant to all mutations were identified as broad neutralizers, however, C3 nAbs with biased neutralization to SARS-CoV-2 showed a reduction of neutralization potencies against mutations in vary degrees (Fig. 1D). Of interesting, the antigenic site consisting of $470-490$ residues (Site $470-490$ ) was determined as a hit area of $\mathrm{C} 3$ nAbs since single mutations within this site significantly decreased the neutralizing activities (Fig. 1D and Fig. S2). The sensitivity of $\mathrm{C} 3 \mathrm{nAbs}$ to E484 residue might cause the diminished neutralization against Beta and Gamma variants. Additionally, there is a substitution of isoleucine for SARS-CoV to phenylalanine for SARS-CoV-2 at position 486 of RBD, the F486I mutation of SARSCoV-2 RBD resulted in the abolish of neutralization of 7 of $11 \mathrm{C} 3$ nAbs (Fig. 1D and Fig. S2), indicating residue 486 play critical role in medicating antigenic diversity between SARS-CoV-2 and SARS-CoV. Taken together, the sequential immunization 
with SARS-CoV and SARS-CoV-2 successfully elicits cross-neutralizing antibodies which are also resistant to all VOCs emerged before Omicron. In addition, the epitopes recognized by $\mathrm{C} 1 \mathrm{nAbs}$ were valuable for deep exploration.

\section{Cross-neutralizing antibodies belong to three clusters with different resistance}

\section{to the Omicron variant}

Since the potently broad neutralization of $\mathrm{C} 1 \mathrm{mAbs}$, it is worthwhile to characterize their function in detail. To this end, an RBD-based competitive binding assay was carried out and those cross-neutralizing antibodies in the C1 class were further divided into three clusters (Fig. 1E). Of all these three clusters of nAbs, representative $n A b$ X17 in Cluster 1 could not block the binding of receptor ACE2 on spike protein, while Cluster 2 and 3 nAbs, represented by X10 and X01, respectively, effectively blocked the binding of ACE2 (Fig. S3). These results suggested that the three clusters of nAbs may recognize three non-overlapping sites on RBD and the epitopes of $\mathrm{X} 01$ (Cluster 3) and X10 (Cluster 2) may overlap to the binding site of ACE2. In addition, the three representative nAbs were determined as potent cross-neutralizing antibodies against both SARS-CoV and SARS-CoV-2 with $I_{50}$ values less than $0.1 \mu \mathrm{g} / \mathrm{mL}$. Remarkedly, X01 showed excellent neutralization, probably due to its effective blocking potency against ACE2 binding (The half-maximal effective concentration $\left(E_{50}\right): 0.17 \mu \mathrm{g} / \mathrm{mL}$ ) (Fig. S3).

We then confirmed the broadly neutralizing efficacies of three representative nAbs against SARS-CoV-2 VOCs including the Omicron variant. X01 and X10 showed 
174

175

potent neutralizing activities with $\mathrm{IC}_{50}$ ranging from 0.04 to $0.16 \mu \mathrm{g} / \mathrm{mL}$ against those VOCs raised between Omicron, which are significantly higher than that of $\mathrm{X} 17\left(\mathrm{IC}_{50}\right.$ ranging from 0.63 to $1.59 \mu \mathrm{g} / \mathrm{mL}$ ) (Fig. 1F). Synchronously, we noted that no point mutations could destroy their neutralization activities, or even reduce $\mathrm{IC}_{50}$ within 10 fold, which further demonstrated the broad neutralizing activities of these three nAbs (Fig. 1D).

The currently dominant VOC, Omicron, which contains unprecedented 15 mutations in RBD, has been shown to be highly resistant to neutralization by plasma from vaccinated individuals, convalescent sera, and most reported neutralizing antibodies (nAbs) ${ }^{11,12}$. We next tested whether cross-neutralizing nAbs $\mathrm{X} 01, \mathrm{X} 10$ and X17 could also potently neutralize Omicron variant. Unfortunately, X01 and X10 remained only weak binding activities against Omicron spike protein with a halfmaximal effective concentration $\left(\mathrm{EC}_{50}\right)$ of 3.29 and $18.79 \mu \mathrm{g} / \mathrm{mL}$, respectively. In contrast, $\mathrm{X} 17$ still maintained strong interaction with the Omicron spike with an $\mathrm{EC}_{50}$ value of $0.005 \mu \mathrm{g} / \mathrm{mL}$ (Fig. 1G). Consistently, X17 showed comparable low neutralizing efficacy $\left(\mathrm{IC}_{50} 3.52 \mu \mathrm{g} / \mathrm{mL}\right.$ ) when compared to WT and other VOCs, while X01 and X10 almost lost their neutralizing activities against the Omicron variant (with IC50 of 49.70 and $50.16 \mu \mathrm{g} / \mathrm{mL}$, respectively). Taken together, the antigenic mutations of the Omicron variant destroyed the neutralizing breadths of nAbs $\mathrm{X} 01$ and $\mathrm{X} 10$ but not $\mathrm{X} 17$.

\section{Cross-neutralizing antibodies define three non-overlapping conserved epitopes} on RBD 
To define the conserved epitopes of the three cross-neutralizing antibodies, we first employed the cryo-EM approach to determine the complex structures of three nAbs combination binding to wild-type spike proteins SARS-CoV-2 (SARS-CoV-2-S) and SARS-CoV (SARS-CoV-S), respectively. Cryo-EM structures of SARS-CoV-2-S and SARS-CoV-S in complex with three nAbs simultaneously were obtained at resolutions of $3.48 \AA$ (Fig. 2A-D, Fig. S4 and 8, Table S2) and $3.83 \AA$ (Fig.2E-G, Fig. S5 and 8, Table S2), respectively. Of interesting, simultaneously binding of three nAbs to SARSCoV-2-S induced the dissociation of trimeric spike and we finally obtained the structure of monomeric spike protein in complex with three nAbs (SARS-CoV-2-S:X01:X10:X17) (Fig. S4). Superimposition of the atomic model of SARS-CoV-2-S:X01:X10:X17 onto the structure of trimeric spike showed conspicuous antibody-induced steric clashes mediated by both $\mathrm{X} 10$ and $\mathrm{X} 17$ but not $\mathrm{X} 01$, suggesting $\mathrm{X} 10$ and $\mathrm{X} 17$ may harbor dissociation potency toward spike trimer (Fig. 2C). Furthermore, the binding of X01 and $X 10$, but not X17, will occupy the space required for ACE2 binding (Fig. 2D), therefore blocking the binding of ACE2 effectively (Fig. S3). As for SARS-CoVS:X01:X10:X17 reconstruction, both trimeric ( 49\%) and dissociated monomer S ( 6\%) in complex with three nAbs were classified out (Fig. S5), suggesting the less potent dissociation efficacy of nAbs on SARS-CoV-S when compared to SARS-CoV-2-S. The particles of trimeric S complexes were selected for further reconstruction (Fig. S5). In the $3.74 \AA$ structure of trimeric SARS-CoV-S:X10:X01:X17, all three RBDs of the spike are in the "up" conformations and bound by three Fabs simultaneously, but only one RBD was saturated bound (Fig. 2G), which was further reconstructed by localized 
refinement to push local resolution (Fig. 2E and Fig. S5). Of note, three RBDs on nAbbound SARS-CoV-S are raised in extremely open states with degrees (compared to close RBD) ranging from 85 to $87^{\circ}$, compared to about $42^{\circ}$ for the normal open RBD in spike protein (Fig. 2H), suggesting that only enough opened RBDs can accommodate three Fabs especially for X10 and X17 to avoid steric clashes and to diminish the disruption of trimeric spike.

We next investigated the simultaneously binding potential of three nAbs to spike proteins of Delta (Delta-S) and Omicron (Omicron-S) variants, respectively. Similar to SARS-CoV-S, the binding of three nAbs on Delta-S induced partial dissociation of trimeric spike (Fig. S6) and we obtained the structures of trimeric Delta-S:X01:X10:X17 at resolutions of $3.54 \AA$ (global refinement) and further performed localized refinement focused on the interface and achieved a structure at $3.77 \AA$ resolution $\quad$ (Fig. 21-K, Fig. S6 and 8, Table S2). As for the Omicron variant, although X01 and X10 showed significantly decreased efficacies in both the binding and neutralizing assays (Fig. 1G and $\mathbf{H})$, we still could obtain a medium resolution (6.56 $\AA$ ) structure of immune complex Omicron-S:X01:X10:X17 (Fig. 2L, Fig. S7 and 8, Table S2).

The footprints of X01, X10 and X17 contain 18, 21 and 16 RBD residues respectively, and only the X10 footprint is found to partially overlap with the ACE2binding site (Y449, Q493) (Fig. 2M-O). The footprints of three nAbs are dramatically non-overlapped between each other and therefore allow the simultaneous binding of three nAbs to RBD (Fig. 2M-P). Furthermore, the footprint of X17 excluded all of the VOCs and VOls mutations (Fig. 20-Q), and the footprints of X10 and X01 contain only 
4 (N440, L452, E484, Q493) and 2 (S371, S375) VOCs mutation sites, respectively (Fig. $2 \mathrm{M}$ and $\mathbf{N}$ ), suggesting their highly conserved epitopes among SARS-CoV-2 VOCs, in particular for that of X17.

\section{Structural basis for the broad neutralization of nAbs against VOCs}

We next analyzed the interaction details of three nAbs to SARS-CoV-2 WT, Delta and SARS-CoV RBDs, respectively. The epitope of X10 in WT RBD composes of 21 residues, including 10 residues (R346, Y351, T345, N440, L441, D442, K444, Y449, N450, T470 and Q493) which form an extensive interaction network containing 15 hydrogen bonds and 1 salt bridges (Fig. 2M, Fig. 3A and B). Although X10 epitope contains VOC mutation site L452 (Fig. 2M), it does not participate in hydrogen bond or salt bridge interaction to X10 (Fig. 3B), the L452R substitution in Delta-RBD not only with no influence on the binding of $X 10$, but instead, the longer side chain of arginine forms additional hydrogen bonds with X10 (Fig. S10B), explaining why X10 confers more than 2-fold higher neutralization against Delta variant $\left(\mathrm{IC}_{50}: 42 \mathrm{ng} / \mathrm{mL}\right)$ than D614G strain (IC $\left.\mathrm{I}_{50}: 86 \mathrm{ng} / \mathrm{mL}\right)$ (Fig. 1F and Table. S1). Additionally, 11 of 20 residues of the X10 epitope in SARS-CoV RBD are conserved among SARS-CoV-2 and SARS-CoV and the other 5 residues are substituted of similar amino acids (Fig. $2 Q$ and Fig. 3C). Consequently, the interactions between the heavy chain of X10 and SARS-CoV RBD are decreased to 3 hydrogen bonds in comparison with 7 hydrogen bonds and 1 salt bridge between X10 and SARS-CoV-2 RBD (Fig. 3A and Fig. S10C). In contrast, the interactions between the light chain of X10 and SARS-CoV RBD are 
enhanced by providing more hydrogen bonds (9 versus 8 ) and salt bridges ( 1 versus 0) compared to that between X10 and SARS-CoV-2 RBD (Fig. 3B and Fig. S10D). Therefore, though $\mathrm{X} 10$ recognizes an epitope that is sequentially diverse between SARS-CoV-2 and SARS-CoV to a certain degree, it still enables it to effectively bind and neutralize SARS-CoV (Fig. 1A and Table S1).

Another cross-neutralizing $\mathrm{mAb}, \mathrm{X} 01$, recognizes a conserved epitope that is near the well-known CR3022 binding site ${ }^{35}$ but a step closer to ACE2 binding site ${ }^{36}$ (Fig. 2N and Fig. S11). Similar to X10, an elaborate interaction network containing 15 hydrogen bonds and 4 salt bridges between $\mathrm{X} 01$ and SASR-CoV-2 RBD occurs in the interface

(Fig. 3D and E). X01 also strongly interacts with Delta RBD and SARS-CoV RBD by providing similar hydrogen bonds and salt bridges interaction as that of WT-RBD (Fig. S10E-H). The epitope of X01 on SARS-CoV RBD comprises 17 residues compared to 18 residues in SARS-CoV-2 RBD, among which, 14 residues are completely conserved (Fig. 2Q and Fig. 3F), which accounts for the comparable neutralizing potencies of X01 against SARS-CoV and SARS-CoV-2 (Fig. 1A and Table S1).

X17 epitope locates at a cryptic site that is far away from the ACE2-binding site, resembles the reported S2H97 26,37 and $6 \mathrm{D} 6 / 7 \mathrm{D} 6^{38}$ (Fig. 20 and Fig. S11A). X17 contacts WT RBD mainly using 9 residues (R355, R357, N394, Y396, D428, K462, F464, E516, H519) by forming an amount of 15 hydrogen bonds and 5 salt bridges (Fig. 3G and H). When binding to Delta RBD and SARS-CoV RBD, the key residues involved in interface interactions are almost unaltered (Fig. S10I-L). Specially, 14 of 16 residues of the epitope of $\mathrm{X} 17$ on SARS-CoV RBD are consistent with that of SARS- 
CoV-2 RBD and the rest 2 residues are substituted by similar amino acid (R357K and K462R) (Fig. 2Q and Fig. 3I). In general, the epitopes of such nAbs that are categorized to Class 5 are highly conserved among Sarbecoviruses and spatially far from almost all the mutation sites of VOCs of SARS-CoV-2 including Omicron variant $^{26,37}$ (Fig. 20-Q, Fig. S11B). Thus, compared to $X 10$ and $X 01, X 17$ is an optimal $\mathrm{nAb}$ with excellent binding activity to Omicron (EC $\left.\mathrm{E}_{50}: 0.005 \mu \mathrm{g} / \mathrm{mL}\right)$ although with unsatisfactory neutralization against Omicron ( $\left.\mathrm{IC}_{50}: 5.7 \mu \mathrm{g} / \mathrm{mL}\right)($ Fig. $1 \mathbf{G}$ and $\mathbf{H})$. As for Omicron, VOC mutation sites E484, Q493 and N440 are involved in X10 interaction while S371 and S375 are involved in X01 interaction (Fig. 2P and Fig. 3J). E484, Q493 and N440 on WT RBD provide appreciable contacts as well as 2 hydrogen bonds interaction with $\mathrm{X} 10$ (Fig. 3K). Although single mutation of any of the three residues was shown not or slightly affect the binding of X10 (Fig. 1D), the synchronal mutation of which may induce the decrease of neutralization of X10 against Omicron. Also, S371 and S375 in WT RBD that included in X01 epitope, involve in appreciable interactions with X01 (Fig. 3J and L). S371 and especially S375 contribute multiple contacts as well as hydrogen bonds interactions between $\mathrm{S} 371$ and $\mathrm{Y} 105^{\mathrm{H}}, \mathrm{S} 375$ and both $\mathrm{Y} 103^{\mathrm{H}}$ and $\mathrm{Y} 105^{\mathrm{H}}$ (Fig. 3L). Therefore, the mutation of these residues causes the devastating decreased neutralizing activity of X01 against Omicron (Fig. 1H). Taken together, the combination of multiple mutations in Omicron RBD makes it easier to escape cross-neutralizing antibodies $\mathrm{X} 10$ and $\mathrm{X} 01$, as well as the majority of existing cross-neutralizing antibodies ${ }^{11}$. In contrast, considering the highly conserved epitope and broadly neutralizing breadth of Class $5 \mathrm{nAb} X 17$, which may serve as an essential 
component of the next-generation antibody cocktail therapeutics against various SARS-CoV-2 variants in the future.

\section{Triple antibody cocktail resists viral escape in vitro}

Several previous studies have reported that combination therapy of dual nAbs targeting noncompeting RBD epitopes decreases rapid viral escape caused by monotherapy ${ }^{13,15,30}$. To understand the escape characteristics of the SARS-CoV-2 under the pressure of each of or cocktail of three nAbs obtained in this study, we performed in vitro escape selection experiments using a previously reported replicative recombinant VSV expressing SARS-CoV-2 spike protein (rVSV-SARS2) ${ }^{30}$ (Fig. S12A). Complete escape of rVSV-SARS2 resistant to X01 or X10 was rapidly selected by 3 passages (Fig. S12B and C). In contrast, X17 could maintain the neutralizing activity over 11 consecutive passages and the completely viral escape was raised at the $13^{\text {th }}$ passage (P13), suggesting that the Class 5 epitope is relatively more tolerant to immunologic pressure (Fig. S12B and C). Furthermore, the triple antibody cocktail showed no viral escape even for 20 passages (Fig. S12B and C). These results indicated that the combination of three cross-neutralizing antibodies composed of X01, $\mathrm{X} 10$ and $\mathrm{X} 17$ has the potential in preventing the rapid emergence of SARS-CoV2 viral escape.

Cross-neutralizing antibody cocktail efficiently protect hamsters from Beta variant infection 
Considering the diverse epitopes, complementary neutralizing breadths and resistance to viral escape of $\mathrm{X} 10, \mathrm{X} 01$ and $\mathrm{X} 17$, we subsequently evaluated the therapeutic activity of triple antibody cocktail against infection of Bata variant (B.1.351) in Syrian hamster model. Following the intranasal challenge of $1 \times 10^{4}$ plaque-forming units (PFU) of B.1.351, antibody cocktail was intravenously administered at a single dose of total $35 \mathrm{mg} / \mathrm{kg}$ (each antibody at $11.7 \mathrm{mg} / \mathrm{kg}$ ) at 1 day post-infection (dpi), then the quantification of viral load and pathological analysis was carried out in the respiratory tract at 5 dpi. Hamsters in the untreated group significantly lost body weight by an average of $13.8 \%$ and $50 \%$ of which were sacrificed at 5 dpi (Fig. 4 A and B). In contrast, hamsters in the treated group showed a more constant weight level with only 2.3\% loss and all survived at $5 \mathrm{dpi}$ (Fig. 4A and B), suggesting the excellent therapeutic efficiency of the triple antibody cocktails.

Next, the viral loads in lung tissues were measured to further evaluate the efficacy of antibody cocktails in the inhibition of viral replication at $5 \mathrm{dpi}$. While the amounts of viral RNA of the untreated group surged to about $1 \times 10^{9}$ copies $/ \mathrm{mL}$ in lung tissues including lung regions proximal (Lu1) and distal (Lu2) to the hilum, hamsters in the antibody-treated group significantly inhibit virus replication by 2-3 order of magnitude in reduction (Fig. 4C). Moreover, in the non-lung respiratory tract, such as nasal turbinates (NT) and trachea (Tr), antibody-treated hamsters were also tested with a significantly decreased viral load when compared to that of untreated hamsters (Fig. 4C). Viral infection-related lung damage was further evaluated. The treatment with antibody cocktail can effectively inhibit the occurrence of multifocal diffuse hyperemia 
and consolidation in gross observations of lung tissues, compared to the untreated group (Fig. S13). Additionally, histopathological examination documented that, compared to the untreated group, there were no significant lesions of alveolar epithelial cells and focal hemorrhage in the lung tissues of hamsters in antibody-treated group (Fig. 4D and E). The treatment with antibody cocktail can profoundly decrease the pathological severity scores to an average of 2.8 , versus 10.7 for the untreated group (Fig. 4F). Collectively, these results revealed that the administration with a triple antibody cocktail can effectively protect hamsters against infection of Bata variant and infection-related lung damage.

\section{Discussion:}

The emerging SARS-CoV-2 variants of concern (VOCs), especially Omicron, showed increased transmissibility and resistance to antibody neutralization and further raised the requirement of broad antibody therapeutics and vaccines ${ }^{11,12,39}$. The recent withdrawal of bamlanivimab has demonstrated that the nAb epitopes located on or adjacent to the RBM under selection pressure are high mutant, and even a combination of two noncompeting nAbs (e.g. bamlanivimab/estevimab) could not prevent the reduction of neutralization potency against SARS-CoV-2 P.1 variant which emerged before Omicron variant ${ }^{40,41}$. It's known that RBD-specific antibodies can be categorized into at least five classes (Class 1 5) based on their binding modes and the competition with ACE2 ${ }^{26}$. Many of reported nAbs from class 3 , class 4 and class 5 , represented by S309 ${ }^{27}$, S2X259 ${ }^{42}$, and S2H97 ${ }^{26,37}$, respectively (Fig. S11B), have 
been demonstrated with broadly neutralizing breadths against many VOCs as well as SARS-CoV. However, most of above nAbs, including those authorized under EUA, decrease their neutralizing activities against Omicron ${ }^{11,12,39,43,44}$. Consistently, crossneutralizing antibodies $\mathrm{X} 01, \mathrm{X} 10$ and $\mathrm{X} 17$ obtained in this study revealed decreased or limited neutralizing potencies against Omicron. According to the previous categorization information, nAbs $\mathrm{X} 10, \mathrm{X} 01$, and $\mathrm{X} 17$ could be classified into Class 3 , Class 4, Class 5, respectively (Fig. S11A). Of note, the binding sites of those rare Class 5 nAbs, were previously revealed spatial cryptic and highly conserved among Sarbecoviruses. The epitope of $\mathrm{X} 17$ revealed in this study confirmed that the binding sites of those Class 5 nAbs are highly conserved between SARS-CoV-2 and SARS$\mathrm{CoV}$, and without any mutations of VOCs including Omicron. Unfortunately, the less potent neutralizing efficacies $\left(\mathrm{IC}_{50}: 1 \sim 10 \mu \mathrm{g} / \mathrm{mL}\right)$ of Class $5 \mathrm{nAbs}$ including $\mathrm{X} 17$ may limit their potential for clinical application. To improve neutralization potency, modification of $\mathrm{X} 17$ volume to strongly block RBD attachment to ACE2 may be a potential optimization direction ${ }^{45,46}$. Nevertheless, the conserved and cryptal epitopes may serve as an ideal target for the development of next-generation broad vaccines against SARS-CoV-2 and variants.

Class C1 antibodies that potently neutralized SARS-CoV and SARS-CoV-2 as well as most VOCs were generated in abundance by sequential immunization with VSV-SARS and VSV-SARS2. We previously have demonstrated that the combined immunization of coronavirus spikes could also effectively elicit cross-neutralizing antibodies ${ }^{38}$. These information together demonstrate that sequential immunization 
can produce cross-neutralizing antibodies targeting to more conserved antigenic sites.

The emergence of the Omicron variant substantially destroy the broad neutralization of most previously reported nAbs that elicit from the infection or immunization of single virus, e.g. SARS-CoV or SARS-CoV-2 prototype strain. Although the Omicron variant generates a big mutational leap on RBD, sequential immunization with VSV-SARS and VSV-SARS2 in this study was showed success to achieve efficient immune focus on those most conserved epitopes, such as epitopes targeted by Class 5 antibodies. However, the mutations of the Omicron variant residing in these conserved epitopes for Class 3 and Class 4 nAbs caused a significant decrease in neutralization potencies. The structural analysis revealed that the E484, Q493 and N440 mutations on Omicron RBD may diminish the binding of Class $3 n A b \times 10$. Based on the previous study, the G446S mutation on Omicron RBD may also diminish the binding of another Class 3 nAb REGN1098747. As for Class 4 nAb X01, S371 and S375 mutations were found to mediate the escape to the Omicron variant. A similar phenomenon may also occur in $\mathrm{H} 014$ by which S371L may mediate the resistance of Omicron to it ${ }^{48}$. Considering the unprecedented evasion from most nAbs and immune response of SARS-CoV-2 prototype strain, Omicron variant should become another important component of sequential immunization, besides SARS-CoV and SARS-CoV-2, to achieve more accurate immune focus, inducing bnAb response against current VOCs and emerging variants in the future.

During the past two years, a large number of antibodies and antibody cocktails have been developed to fight COVID-19. In this study, we evaluated the combination 
of three cross-neutralizing nAbs against SARS-CoV-2 infection in animals for the first time. $\mathrm{X} 01, \mathrm{X} 10$ and $\mathrm{X} 17$ were identified to effectively protect against B.1.351 infection in vivo. A cocktail of three mAbs (atoltivimab, maftivimab, and odesivimab) combatting Ebola virus have been approved in $2020^{49}$. These results provide important insights into the feasibility for the development of a triple antibody cocktail against infectious diseases. Structure analyses revealed the existence of at least three noncompeting cross-neutralizing epitopes arraying around RBD. The simultaneously occupying of such epitopes, e.g. simultaneously binding of $X 01, X 10$ and $X 17$, will shield the majority of the flank, therefore performing excellent or even synergetic neutralization against SARS-CoV-2 and variants. For this triple antibody cocktail, $\mathrm{X} 01$ and $\mathrm{X} 10$ moderately block RBD binding to ACE2 and confer potent neutralization $\left(\mathrm{IC}_{50}: 0.05-0.16 \mu \mathrm{g} / \mathrm{mL}\right)$ against SARS-CoV-2 and most VOCs mainly by interfering virus-receptor interaction. The third nAb X17, which recognized the highly conserved and cryptic epitope with an excellent binding affinity, buried in the lying down RBD similar to that CR3022-like antibodies50, may further neutralize virus by destabilization of spike protein. Furthermore, although less potent when compared to the of single nAbs against other VOCs, the triple antibody cocktail showed a synergetic neutralizing efficacy $\left(\mathrm{IC}_{50}: 3.5\right.$ $\mu \mathrm{g} / \mathrm{mL}$ ) against Omicron. Considering that the clinical administration antibody therapeutics always use an extremely large dosage, e.g. Trump received antibody treatment for COVID-19 with total dosage of $8 \mathrm{~g}^{6}$. The $3.5 \mu \mathrm{g} / \mathrm{mL}$ level of neutralization may also provide effective protection from Omicron infection, which will be confirmed in our further animal studies. Additionally, previous studies have demonstrated a higher 
frequency of mutations in the RBM over the rest of the RBD ${ }^{51}$. The footprints of three nAbs are with no or few overlaps to the ACE2 binding site, which may further benefit in avoiding antibody-induced immune escape. Indeed, the absence of escape variants under the pressure of a single $\mathrm{X} 17$ further implies that escape mutations residing in the X17 epitope might destroy SARS-CoV-2 replication, possibly due to the important role of this epitope in the SARS-CoV-2 infection process. The triple antibody cocktail based on X17 provides stronger prevention against a selection of rapid escape viruses, resulting from the synergistic inhibition that is lacking for single antibody therapeutics. These results demonstrated that the triple antibody cocktail is a promising candidate for immunotherapy against pandemic SARS-CoV-2 VOCs.

In summary, the above results documented that sequential immunization could achieve immune focus on the conserved epitopes of SARS-CoV and SARS-CoV-2 to induce amounts of bnAbs against SARS-CoV, SARS-CoV-2 and VOCs. The combination of three representative bnAbs $\mathrm{X} 01, \mathrm{X} 10$ and $\mathrm{X} 17$ exhibited synergistic neutralizing activities, resistance to viral escape and protection of hamsters from disease caused by SARS-CoV-2. We also define the structural basis for neutralization breadth and potency by this triple antibody cocktail. This study suggests a new strategy for the development of antibody therapeutics as well as universal SARS-CoV-2 vaccines based on immune focus.

\section{Methods}

\section{Ethics statement}


461

Ltd. Hamsters (LVG golden Syrian hamsters) were purchased from Charles River Laboratories. All experiments with infectious SARS-CoV-2 were performed in the biosafety level 3 (BSL-3) and animal biosafety level 3 (ABSL-3) facilities. The Golden Syrian Hamster (Charles River Laboratories) was raised in specific pathogen-free animal feeding facilities. All animal studies were carried out in strict accordance with the recommendations of the Guide for the Care and Use of Laboratory Animals. The mouse and hamster studies were conducted under the approval of the Institutional Animal Care and Use Committee of Xiamen University. All the animal experiments were approved by the Medical Ethics Committee (SUCM2021-112).

\section{Cell lines}

Vero-E6 (American Type Culture Collection [ATCC], CRL-1586), BHK21 (ATCC, CCL-10), SP2/0 (ATCC), and 293T (kindly gifted by Dr. Jiahuai Han) cells were maintained in high glucose DMEM (SIGMA-ALDRICH) supplemented with 10\% FBS (GIBCO), penicillin (100 IU/mL), streptomycin $(100 \mu \mathrm{g} / \mathrm{mL})$ in a $5 \% \mathrm{CO} 2$ environment at $37^{\circ} \mathrm{C}$ and passaged every 2 days. BHK21-hACE2 cell was developed by stable transfection of hACE2-expressing plasmid following puromycin resistance selection. All cell lines used in this study were routinely tested for mycoplasma and found to be mycoplasma-free.

\section{Production of pseudoviruses}

Recombinant vesicular stomatitis virus (rVSV) expressing SARS-CoV spike(GenBank: AY278554.2) (termed as rVSV-SARS), SARS-CoV-2 prototype strain 
482

483

spike (GenBank: MN908947) (termed as rVSV-SARS2), SARS-CoV-2 VOCs spikes or 55 SARS-CoV-2 spikes with different single point mutations were generated as previously described ${ }^{34}$. Briefly, the spike gene of SARS-CoV or SARS-CoV-2 with a Cterminal 18 amino acids truncation was cloned into the eukaryotic expression plasmid pCAG (Addgene), respectively. rSARS-CoV and rSARS-CoV2 were rescued by VSVdG-EGFP-G (Addgene, 31842) from the Vero E6 cells transfected with plasmids pCAG-SARS1-Sdel18 and pCAG-SARS2-Sdel18 $8^{52}$, respectively. The supernatant was harvested and purified by Capto Core 700 (Cytiva) multimodal chromatography, the viral particles were collected in the column flowthrough.

\section{Neutralization assay based on VSV pseudovirus}

Neutralizing activities of antibodies against SARS-CoV, SARS-CoV-2, SARSCoV-2 VOCs and SARS-CoV-2 with different single point mutations were quantified based on recombinant VSV as previously described ${ }^{34}$, a series of diluted monoclonal antibodies were mixed with pseudoviruses carrying spike of SARS-CoV, SARS-CoV-2 or variants and incubated at $37^{\circ} \mathrm{C}$ for 1 hour, respectively. Then the mixture was transferred to BHK21-hACE2 cells seeded in 96-well microplate. After 12 hours of incubation, fluorescence images were captured by Opera Phenix (PerkinElmer) and quantitatively analyzed by the Columbus system (PerkinElmer). The reduction percentage of EGFP in each well compared to the control wells was calculated. The $\mathrm{IC}_{50}$ value was determined by the 4-parameter logistic regression using GraphPad Prism (version 8.0.1).

\section{Sequential immunization and cross-neutralizing antibody screening}


a week, alternately. After six doses of immunization, mice were sacrificed and the

spleen cells were harvested for cell fusion with mouse myeloma cell line SP2/0 to generate hybridomas. The pseudovirus neutralization assay based on rVSV-SARS and rVSV-SARS2 were used to screen the hybridomas secreting cross-neutralizing antibody against both SARS-CoV and SARS-CoV-2. The screening assay was described previously ${ }^{34}$.

\section{Blocking capacity of nAbs against ACE2 binding}

Microplates pre-coated with recombinant antigens of RBD were provided by the

Wantai BioPharm. Antibodies at $100 \mu \mathrm{g} / \mathrm{mL}$, respectively, were five-fold serially diluted, added to the wells $(100 \mu \mathrm{L})$, and incubated at $37^{\circ} \mathrm{C}$ for $0.5 \mathrm{~h}$. ACE2-hFc (provided by the Wantai BioPharm) was diluted at $85 \mathrm{ng} / \mathrm{mL}$ in SD-1 (Wantai BioPharm), added to the wells $(100 \mu \mathrm{L})$, and incubated at $37^{\circ} \mathrm{C}$ for $0.5 \mathrm{~h}$. Then wells were washed, $\mathrm{A}$ horseradish peroxidase (HRP)-labeled goat anti-human antibody (Abcam) was used as the secondary antibody at $1: 5000$ for 30 min. Wells were washed again and the reaction was catalyzed using o-phenylenediamine substrate at $37^{\circ} \mathrm{C}$ for $10 \mathrm{~min}$. The OD450 nm (reference, OD630nm) was measured on a microplate reader (TECAN,

522 measured quantitatively by comparing OD in the presence and absence of nAbs, and

523 transformed using the formula $\left[1-\left(O D_{\text {present }} / O D_{\text {absent }}\right)\right] \times 100 \%$. and the blocking $I_{50}$ 524 values were calculated by Prism software using non-linear regression (four 525 parameters). 


\section{Competition binding assay}

Briefly, the unlabeled nAbs (50 $\mu \mathrm{g}$ per well) or $20 \mathrm{mM}$ phosphate-buffered saline (PBS) (GIBCO) were added to SARS-CoV-2 RBD-coated 96-well microplates and then incubated for $30 \mathrm{~min}$ at $37^{\circ} \mathrm{C}$. Next, HRP-conjugated nAbs were added at selected dilutions, at which OD readings was $\sim 1.5$ present. After incubation for $30 \mathrm{~min}$ at $37^{\circ} \mathrm{C}$, the microplates were rinsed and the color was developed. The blocking rate was measured quantitatively by comparing OD in the presence and absence of competitor mAbs, and transformed using the formula $\left[1-\left(O D_{\text {present }} / O D_{\text {absent }}\right)\right] \times 100 \%$.

\section{Cryo-EM sample and data collection}

Aliquots $(3 \mu \mathrm{L})$ of $3.5 \mathrm{mg} / \mathrm{mL}$ mixtures of purified SARS-CoV-2 WT-S, Delta-S, Omicron-S proteins (Sino Biological Inc.) and SARS-CoV-S protein (Sino Biological Inc.) in complex with excess Fab fragments of three nAbs were incubated in $0.01 \%$ (v/v) Digitonin (Sigma) and then loaded onto glow-discharged (60 s at $20 \mathrm{~mA}$ ) holey carbon Quantifoil grids (R1.2/1.3, 200 mesh, Quantifoil Micro Tools) using a Vitrobot Mark IV (ThermoFisher Scientific) at $100 \%$ humidity and $4^{\circ} \mathrm{C}$. Data were acquired using the SerialEM software on an FEI Tecnai F30 transmission electron microscope (ThermoFisher Scientific) operated at $300 \mathrm{kV}$ and equipped with a Gatan K3 direct detector. Images were recorded in the 36 -frame movie mode at a nominal $39,000 \times$ magnification at super-resolution mode with a pixel size of $0.389 \AA$. The total electron dose was set to $60 \mathrm{e}^{-} \AA^{-2}$ and the exposure time was $4.5 \mathrm{~s}$.

\section{Image processing and 3D reconstruction}

Drift and beam-induced motion correction were performed with MotionCor2 ${ }^{53}$ to 
produce a micrograph from each movie. Contrast transfer function (CTF) fitting and phase-shift estimation were conducted with Gctf $^{54}$. Micrographs with astigmatism, obvious drift, or contamination were discarded before reconstruction. The following reconstruction procedures were performed by using Cryosparc V $3^{55}$. In brief, particles were automatically picked by using the "Blob picker" or "Template picker". Several rounds of reference-free 2D classifications were performed and the selected good particles were then subjected to ab-initio reconstruction, heterogeneous refinement and final non-uniform refinement. The resolution of all density maps was determined by the gold-standard Fourier shell correlation curve, with a cutoff of $0.143^{56}$. Local map resolution was estimated with ResMap $^{57}$.

\section{Atomic model building, refinement, and 3D visualization}

The initial model of nAbs was generated from homology modeling by Accelrys Discovery Studio software (available from: URL: https://www.3dsbiovia.com). The structure of SARS-CoV-2 RBD and SARS-CoV RBD from the structure of WT trimeric spike (pdb no. 6VSB ${ }^{58}$ ) and SARS-CoV RBD in complex with CR3022 (pdb no. $7 \mathrm{JN} 5^{59}$ ), respectively, were used as the initial modes of our WT-RBD,Delta-RBD and SARS-CoV RBD. We initially fitted the templates into the corresponding final cryo-EM maps using Chimera ${ }^{60}$, and further corrected and adjusted them manually by realspace refinement in $\operatorname{Coot}^{61}$. The resulting models were then refined with phenix.real_space_refine in PHENIX ${ }^{62}$. These operations were executed iteratively until the problematic regions, Ramachandran outliers, and poor rotamers were either eliminated or moved to favored regions. The final atomic models were validated with 
Molprobity ${ }^{63,64}$. All figures were generated with Chimera or ChimeraX ${ }^{65,66}$.

The initial model of nAbs was generated from homology modeling by Accelrys Discovery Studio software (available from: URL: https://www.3dsbiovia.com). The structure of RBD from the structure of WT trimeric spike (pdb no. $6 \mathrm{VSB}^{58}$ ) was used as the initial modes of our WT-RBD and Omicron RBD. We initially fitted the templates into the corresponding final cryo-EM maps using Chimera ${ }^{60}$, and further corrected and adjusted them manually by real-space refinement in $\operatorname{Coot}^{61}$. The resulting models were then refined with phenix.real_space_refine in PHENIX ${ }^{62}$. These operations were executed iteratively until the problematic regions, Ramachandran outliers, and poor rotamers were either eliminated or moved to favored regions. The final atomic models were validated with Molprobity ${ }^{63,64}$. All figures were generated with Chimera or ChimeraX ${ }^{65,66}$.

\section{Generation of replicative recombinant VSV-SARS2 virus}

Replicative recombinant VSV-SARS2 (rrVSV-SARS2) was generated by replacing the VSV glycoprotein with the native SARS-CoV-2 spike protein from WuhanHu-1 strain (GenBank: MN908947) with a C-terminal 18 amino acids truncation and encoding the GFP genes insert to 3' end of VSV genome. 293T cells were plated on Poly-L-lysine solution (SIGMA-ALDRICH) treated plates and incubated overnight in DMEM (SIGMA-ALDRICH) containing 10\% fetal bovine serum (GIBCO) and $1 \%$ Penicillin/Streptomycin/L-glutamine (Invitrogen). The following day, the cells were infected by recombinant vaccinia virus producing the T7 RNA polymerase (rVV-T7) and transfected with the VSV genomic clone driven by a T7 promoter and helper plasmids 
592 593 (Invitrogen). After 48 hours, the supernatant of transfected cells was co-cultured with 594 Vero E6 cells (ATCC) transfected with VSV-G. Cells were monitored for GFP 595

expressing the VSV-N, VSV-P, VSV-G, VSV-L with Lipofectamine LTX reagent expression or cytopathic effect (CPE) indicative of virus replication. Virus was then expanded and titered in BHK21-hACE2 cells. After collection, stocks of both viruses were centrifuged at $3500 \mathrm{rpm}$ for 5 minutes to clarify and frozen at $-80^{\circ} \mathrm{C}$.

\section{In vitro escape studies}

Escape studies were performed with rrVSV-SARS2 virus ${ }^{30}$. Viral escape was selected by incubating rrVSV-SARS2 under antibody pressure ranging from 0.02 $\mu \mathrm{g} / \mathrm{mL}$ to $20 \mu \mathrm{g} / \mathrm{mL}$. After 60 minutes of incubation, the mixture was used to infect $1 \times 10^{6}$ Vero E6 cells at a multiplicity of infection (MOI) of 1 . Virus replication was monitored by screening for GFP expression or cytopathic effect (CPE) over 96 hours. When $>90 \%$ cells were GFP-positive or exhibit $90-100 \%$ CPE, the supernatant was collected and clarified by centrifugation. For subsequent rounds of selection, $100 \mu \mathrm{L}$ of supernatant containing the virus was passaged under the same or greater antibody concentrations as in previous passages until complete CPE was observed after antibody treatment at a concentration of $\geqslant 20 \mu \mathrm{g} / \mathrm{mL}$. The consecutive passages virus was then expanded and titered in BHK21-hACE2 cells. Neutralization assays of antibodies against the virus consecutive passaged were made as previously described ${ }^{34}$.

\section{Therapeutic effects against Beta variant in hamsters}

The therapeutic effects of cross-neutralizing antibodies cocktail against Beta variant (GISAID: EPI_ISL_2779639) was evaluated using a hamster model as 
614

previously descripted ${ }^{67}$. Briefly, hamsters were intranasally inoculated with $1 \times 10^{4}$ PFU/100 $\mu$ L of SARS-CoV-2 Beta strain. The triple antibody cocktail composed of X01, $\mathrm{X} 10$ and $\mathrm{X} 17$ in a ratio of 1:1:1 was administrated intraperitoneally at a total dose of $35 \mathrm{mg} / \mathrm{kg}$ at 24 hours post-challenge, PBS was used as negative control. The body weight change and health status were recorded daily. Hamsters were euthanized at 6 days post-challenge for detection of viral load in respiratory tract organs and analysis of pathogenesis in lung lobes. The indicators of therapeutic efficacy were including body weight change, tissue viral RNA load, and the histopathology examination score were tested.

\section{SARS-CoV-2 RNA quantification}

The tissue samples including lung, trachea and nasal turbinate were separated from infected hamsters and homogenized with TissueLyser II (Qiagen), and SARSCoV-2 RNA was extracted using the QIAamp Viral RNA Mini Kit ((52906, Qiagen). Then, the viral RNA concentration was quantified using a SARS-CoV-2 RT-PCR Kit (WS-1248, Wantai BioPharm) according to the manufacturer's instructions.

\section{Quantification and statistical analysis}

GraphPad Prism (version 8.0.1) was used for all statistical calculations. To compare continuous variables, Mann Whitney test was performed between groups. For statistical difference analysis, $\mathrm{P}$ values less than 0.05 were considered statistically significant. ns: not significant; ${ }^{*} \mathrm{P}<0.05 ;{ }^{* *} \mathrm{P}<0.01$; ${ }^{* * *} \mathrm{P}<0.001$. IC $\mathrm{I}_{50}$ values were calculated by non-linear regression analysis (log(agonist) vs response - Variable slope (four parameters)). 
636

637 
638

639

640

641

642

643

644

645

646

647

648

649

650

651

652

653

654

655

656

657

658

659

Acknowledgments: This study was supported by the National Natural Science Foundation of China (81991491 (to N.X.), 31730029 (to N.X.), 32170943 (to T.Z.), 82001756 to T.L.), Fujian Natural Science Foundation for Distinguished Young Scholars (2020J06007 (to T.Z.)), Xiamen Youth Innovation Fund Project (3502Z20206060 (to T.Z)) and the Bill \&Melinda Gates Foundation (INV-005834 (to N.X.)).

Author contributions: N.X., T.Z., Q.Z., J.Z. and H.X. designed the study; Y.Z., J.Z., Y.J., M.W. and Y.W. participated in the neutralization assay; L.M., S.Y. and Y.W performed the viral escape assay; J.M., M.Z., L.Y., T.C. and Y.G. designed and performed the therapy experiment in the hamster model; R.F. and M.Y. tested the binding activity; H.S., L.L. and Y.H. prepared the cryo-EM grids and recorded the cryoEM movies; H.S., T.L. and Q.Z. processed the data and obtained all 3D reconstructions; N.X., T.Z., H.X., R.Q. and S.W. analyzed data; H.X., H.S., S.W. and T.Z. wrote the manuscript; Q.Y., Z.Z., S.L., S.W., T.Z., Y.G., Q.Z. and N.X. participated in the discussion and interpretation of the results. All authors reviewed and approved the paper.

Data availability: The cryo-EM density maps have been deposited in the Electron Microscopy Data Bank (EMDB) with the accession codes of $X X X, X X X, X X X, X X X$, $\mathrm{XXX}$, and the corresponding atomic coordinates have been deposited in the Protein Data Bank (PDB) with the accession codes of XXX, XXX, XXX, XXX, XXX.

Conflict of interest: The authors declare that they have no conflicts of interest. 


\section{References:}

6611 Zhou, P. et al. A pneumonia outbreak associated with a new coronavirus of probable bat origin.

662

6632

664

6653

666

6674

668

6695

670

$671 \quad 6$

672

6737

$674 \quad 8$

675

$676 \quad 9$

677

$678 \quad 10$

679

$680 \quad 11$

681

$682 \quad 12$

683

$684 \quad 13$

685

$686 \quad 14$

687

688

$689 \quad 15$

690

69116

692

$693 \quad 17$

694

69518

696

697

698

69920

700

701

$702 \quad 21$

703

Nature 579, 270-273, doi:10.1038/s41586-020-2012-7 (2020).

Burki, T. Understanding variants of SARS-CoV-2. Lancet 397, 462, doi:10.1016/S01406736(21)00298-1 (2021).

Wang, C., Horby, P. W., Hayden, F. G. \& Gao, G. F. A novel coronavirus outbreak of global health concern. Lancet 395, 470-473, doi:10.1016/S0140-6736(20)30185-9 (2020).

Barnes, C. O. et al. SARS-CoV-2 neutralizing antibody structures inform therapeutic strategies. Nature 588, 682-687, doi:10.1038/s41586-020-2852-1 (2020).

Ju, B. et al. Human neutralizing antibodies elicited by SARS-CoV-2 infection. Nature 584, 115119, doi:10.1038/s41586-020-2380-z (2020).

Tomas, K. K., G. President Trump Received Experiment Antibody Treatment, <https://www.nytimes.com/2020/10/02/health/trump-antibody-treatment.html> (2020).

An EUA for sotrovimab for treatment of COVID-19. Med Lett Drugs Ther 63, 97-xx98 (2021).

An EUA for Bamlanivimab-A Monoclonal Antibody for COVID-19. JAMA 325, 880-881, doi:10.1001/jama.2020.24415 (2021).

Casirivimab and imdevimab (REGEN-COV) for post-exposure prophylaxis of COVID-19. Med Lett Drugs Ther 63, 130-131 (2021).

Dougan, M. et al. Bamlanivimab plus Etesevimab in Mild or Moderate Covid-19. N Engl J Med 385, 1382-1392, doi:10.1056/NEJMoa2102685 (2021).

Cao, Y. et al. Omicron escape the majority of existing SARS-CoV-2 neutralizing antibodies. Nature, doi:10.1038/d41586-021-03796-6 (2021).

Cele, S. et al. Omicron extensively but incompletely escapes Pfizer BNT162b2 neutralization. Nature, doi:10.1038/d41586-021-03824-5 (2021).

Baum, A. et al. Antibody cocktail to SARS-CoV-2 spike protein prevents rapid mutational escape seen with individual antibodies. Science 369, 1014-+, doi:10.1126/science.abd0831 (2020).

Liu, Z. M. et al. Identification of SARS-CoV-2 spike mutations that attenuate monoclonal and serum antibody neutralization. Cell Host Microbe 29, 477-+, doi:10.1016/j.chom.2021.01.014 (2021).

5 Weisblum, Y. et al. Escape from neutralizing antibodies by SARS-CoV-2 spike protein variants. Elife 9, doi:ARTN e6131210.7554/eLife.61312 (2020).

6 Wang, P. F. et al. Antibody resistance of SARS-CoV-2 variants B.1.351 and B.1.1.7. Nature 593, 130-+, doi:10.1038/s41586-021-03398-2 (2021).

Hoffmann, M. et al. SARS-CoV-2 variants B.1.351 and P.1 escape from neutralizing antibodies. Cell 184, 2384-+, doi:10.1016/j.cell.2021.03.036 (2021).

18 Wang, P. F. et al. Increased resistance of SARS-CoV-2 variant P.1 to antibody neutralization. Cell Host Microbe 29, 747-+, doi:10.1016/j.chom.2021.04.007 (2021).

19 Li, Q. Q. et al. The Impact of Mutations in SARS-CoV-2 Spike on Viral Infectivity and Antigenicity. Cell 182, 1284-+, doi:10.1016/j.cell.2020.07.012 (2020).

20 Starr, T. N., Greaney, A. J., Dingens, A. S. \& Bloom, J. D. Complete map of SARS-CoV-2 RBD mutations that escape the monoclonal antibody LY-CoV555 and its cocktail with LY-CoV016. Cell Rep Med 2, 100255, doi:10.1016/j.xcrm.2021.100255 (2021).

21 Hoffmann, M. et al. SARS-CoV-2 Cell Entry Depends on ACE2 and TMPRSS2 and Is Blocked by a Clinically Proven Protease Inhibitor. Cell 181, 271-+, doi:10.1016/j.cell.2020.02.052 (2020). 


5

Matheson, N. J. \& Lehner, P. J. How does SARS-CoV-2 cause COVID-19? Science 369, 510-511, doi:DOI: 10.1126/science.abc6156 (2020).

$\mathrm{Wu}, \mathrm{Y}$. et al. A noncompeting pair of human neutralizing antibodies block COVID-19 virus binding to its receptor ACE2. Science 368, 1274-+, doi:10.1126/science.abc2241 (2020).

Piccoli, L. et al. Mapping Neutralizing and Immunodominant Sites on the SARS-CoV-2 Spike Receptor-Binding Domain by Structure-Guided High-Resolution Serology. Cell 183, 1024-+, doi:10.1016/j.cell.2020.09.037 (2020).

Cao, Y. L. et al. Humoral immune response to circulating SARS-CoV-2 variants elicited by inactivated and RBD-subunit vaccines. Cell Res 31, 732-741, doi:10.1038/s41422-021-00514-9 (2021).

7

Starr, T. N. et al. SARS-CoV-2 RBD antibodies that maximize breadth and resistance to escape. Nature 597, 97-102, doi:10.1038/s41586-021-03807-6 (2021).

Nature 583, 290-295, doi:10.1038/s41586-020-2349-y (2020).

Tortorici, M. A. et al. Broad sarbecovirus neutralization by
Nature 597, 103-+, doi:10.1038/s41586-021-03817-4 (2021).

9 Starr, T. N. et al. SARS-CoV-2 RBD antibodies that maximize breadth and resistance to escape. Nature 597, 97-+, doi:10.1038/s41586-021-03807-6 (2021).

Copin, R. et al. The monoclonal antibody combination REGEN-COV protects against SARS-CoV2 mutational escape in preclinical and human studies. Cell 184, 3949-3961 e3911, doi:10.1016/j.cell.2021.06.002 (2021).

1 Shen, C. et al. A multimechanistic antibody targeting the receptor binding site potently crossprotects against influenza B viruses. Sci Transl Med 9, doi:10.1126/scitranslmed.aam5752 (2017).

2 Skowronski, D. M. et al. Cross-lineage influenza B and heterologous influenza A antibody responses in vaccinated mice: immunologic interactions and $\mathrm{B} /$ Yamagata dominance. PLoS One 7, e38929, doi:10.1371/journal.pone.0038929 (2012).

Maroof, A., Yorgensen, Y. M., Li, Y. \& Evans, J. T. Intranasal vaccination promotes detrimental Th17-mediated immunity against influenza infection. PLoS Pathog 10, e1003875, doi:10.1371/journal.ppat.1003875 (2014).

Xiong, H. L. et al. Robust neutralization assay based on SARS-CoV-2 S-protein-bearing vesicular stomatitis virus (VSV) pseudovirus and ACE2-overexpressing BHK21 cells. Emerg Microbes Infect 9, 2105-2113, doi:10.1080/22221751.2020.1815589 (2020).

Yuan, M. et al. Structural basis of a shared antibody response to SARS-CoV-2. Science 369, 11191123, doi:10.1126/science.abd2321 (2020). Yan, R. et al. Structural basis for the recognition of SARS-CoV-2 by full-length human ACE2. Science 367, 1444-1448, doi:10.1126/science.abb2762 (2020).

Cameroni, E. et al. Broadly neutralizing antibodies overcome SARS-CoV-2 Omicron antigenic shift. Nature, doi:10.1038/s41586-021-04386-2 (2021).

Li, T. et al. Cross-neutralizing antibodies bind a SARS-CoV-2 cryptic site and resist circulating variants. Nat Commun 12, 5652, doi:10.1038/s41467-021-25997-3 (2021).

Cameroni, E. et al. Broadly neutralizing antibodies overcome SARS-CoV-2 Omicron antigenic shift. Nature, doi:10.1038/d41586-021-03825-4 (2021).

Update on COVID-19 variants and impact on bamlanivimab distribution., 


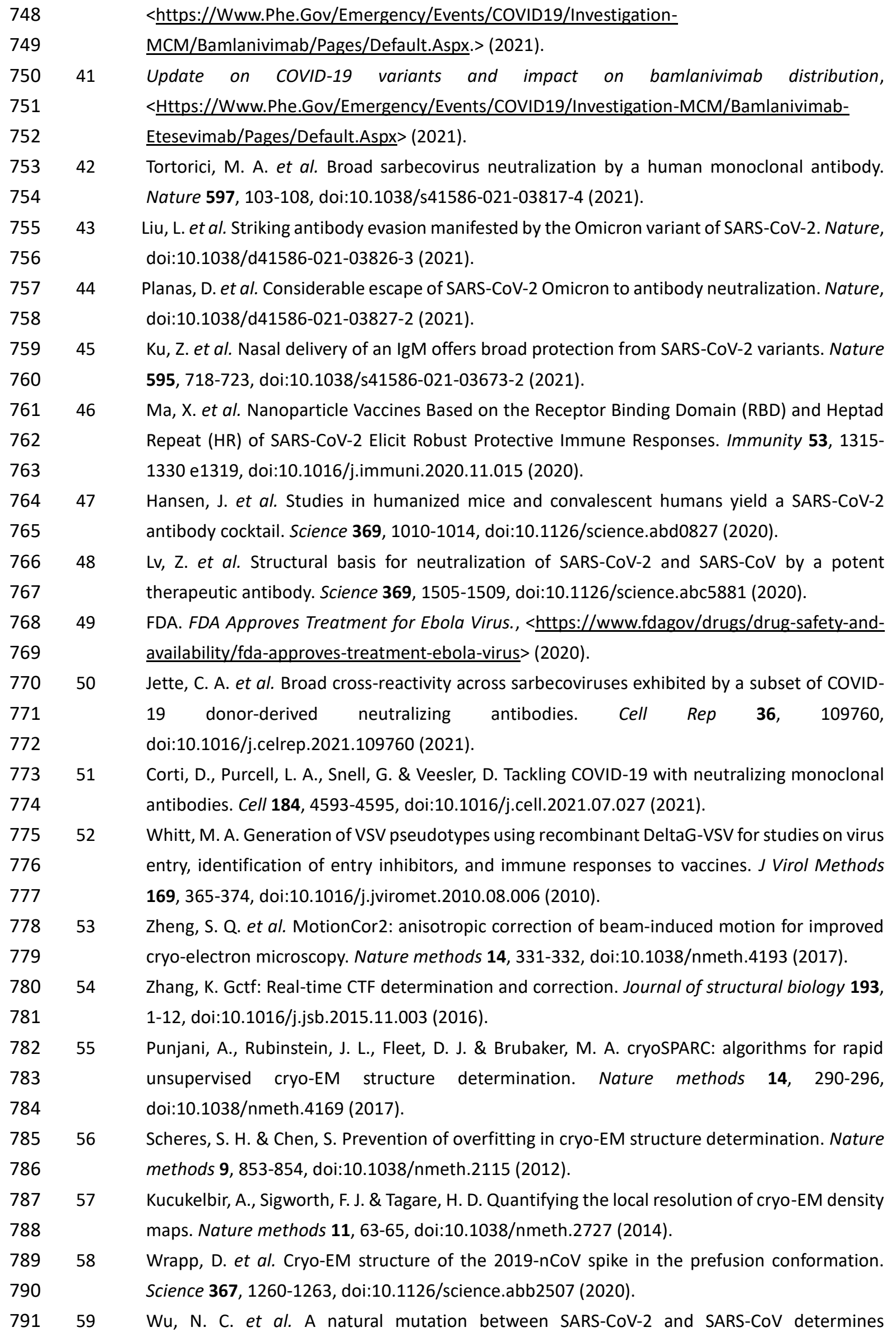


neutralization by a cross-reactive antibody. PLoS Pathog 16, e1009089, doi:10.1371/journal.ppat.1009089 (2020).

60 Pettersen, E. F. et al. UCSF Chimera--a visualization system for exploratory research and analysis. Journal of computational chemistry 25, 1605-1612, doi:10.1002/jcc.20084 (2004). Emsley, P. \& Cowtan, K. Coot: model-building tools for molecular graphics. Acta crystallographica.

\author{
Section
}

D, Biological

crystallography

60, 2126-2132, doi:10.1107/S0907444904019158 (2004).

800

Adams, P. D. et al. PHENIX: a comprehensive Python-based system for macromolecular

801

802 structure solution. Acta crystallographica. Section D, Biological crystallography 66, 213-221, doi:10.1107/S0907444909052925 (2010).

803

804

805

Chen, V. B. et al. MolProbity: all-atom structure validation for macromolecular crystallography.

806

807

Acta crystallographica. Section doi:10.1107/S0907444909042073 (2010).

808

$809 \quad 66$

810

811

67

812

813

814 

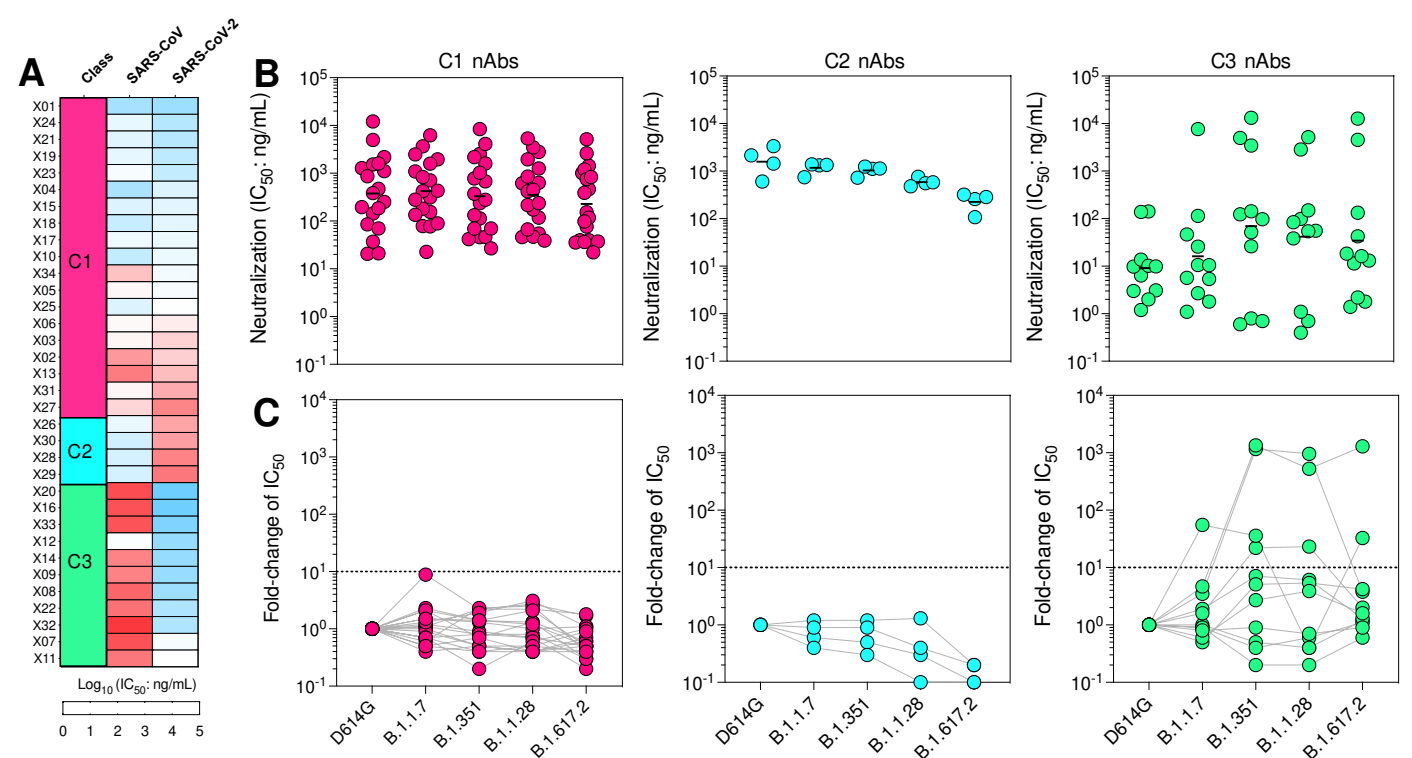

D
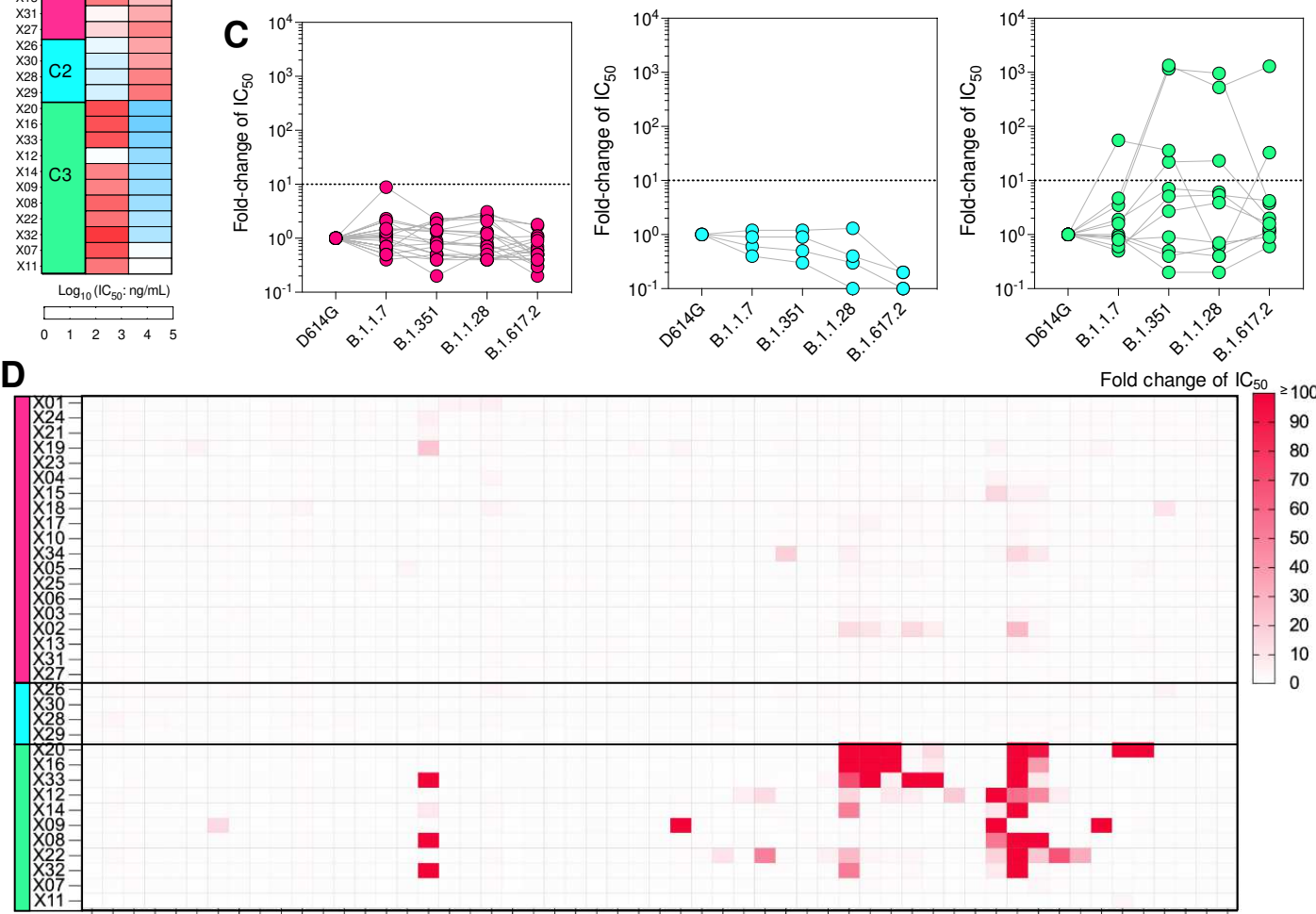

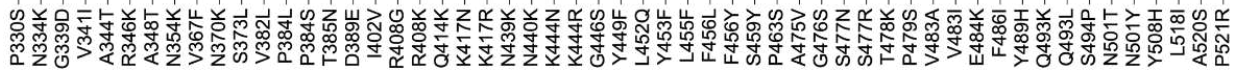
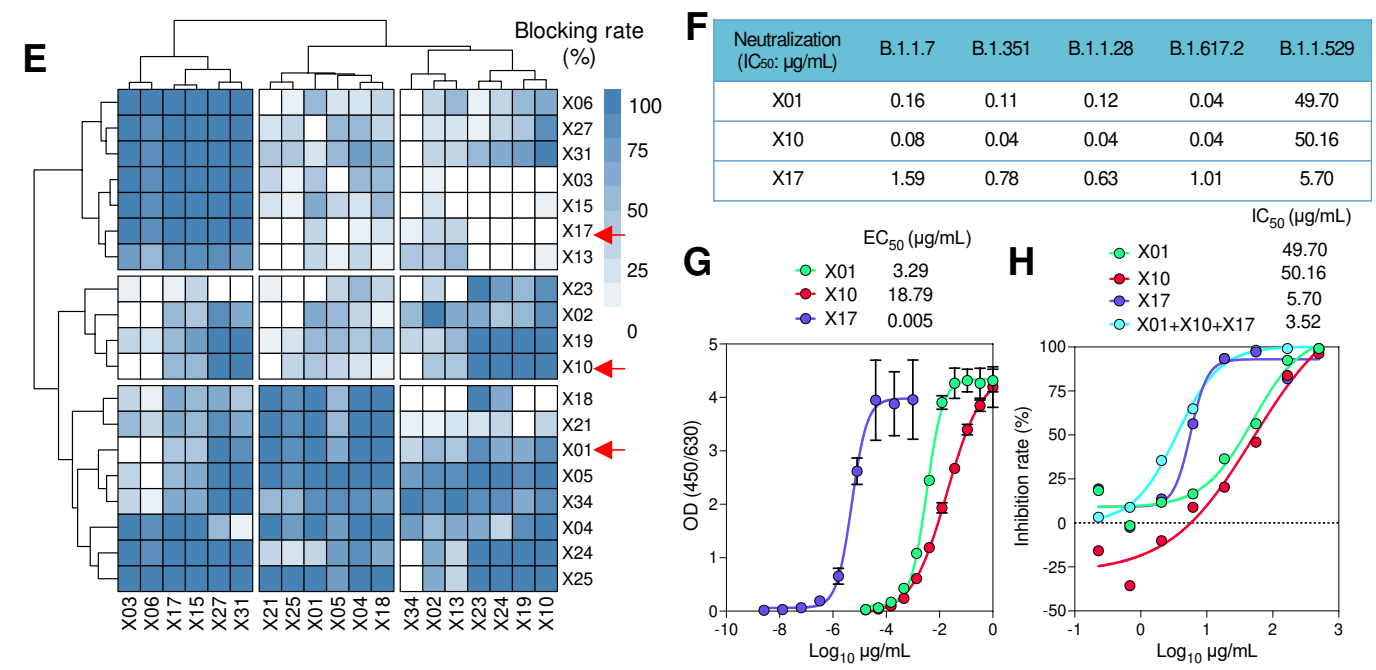

816 Fig. 1. Characterization of broadly neutralizing nAbs induced by sequential immunization of pseudoviruses of SARS-CoV and SARS-CoV-2. (A) Classification 
of a panel of 34 nAbs based on their cross-neutralization of SARS-CoV and SARSCoV-2. The neutralization potencies of nAbs were evaluated by recombinant VSVbased pseudoviruses of SARS-CoV and SARS-CoV-2. The $\mathrm{IC}_{50}$ values ranging from 1 $\mathrm{ng} / \mathrm{mL}$ to $50 \mu \mathrm{g} / \mathrm{mL}$ were represented in blue to red, respectively. nAbs were classified into classes $C 1, C 2$ and $C 3$, based on the fold change of $I C_{50}$ values against SARSCoV-2 related to SARS-CoV (class C1: 0.1-10, class C2: $<0.1$, and class C3: $>10)$. (B and C) The $I C_{50}$ values of three classes of $n A b s$ against pseudoviruses of D614G strain and VOCs B.1.1.7, B.1.351, B.1.1.28 and B.1.617.2 (B) and the $\mathrm{IC}_{50}$ fold change compared to that of prototyped SARS-CoV-2 (C). nAbs of Classes C1, C2 and C3 are colored in magenta, cyan and green, respectively. The black lines in (B) indicate the geometric means and the black dashed lines in (C) indicate 10 times in fold change. (D) Interfere of single point mutations on neutralization potencies of nAbs in classes $\mathrm{C} 1, \mathrm{C} 2$ and $\mathrm{C} 3$. The $\mathrm{IC}_{50}$ values for different $\mathrm{nAbs}$ were determined against VSV pseudovirus carrying SARS-CoV-2 S protein with single residue substations. The fold changes of $I C_{50}$ values of these mutant pseudoviruses (related to D614G control) were calculated. The abscissa shows different mutant residues. (E) Cross-blocking matrix for class $\mathrm{C} 1 \mathrm{nAbs}$. The concentrations of blocking nAbs (row) and detective nAbs (column) were $500 \mu \mathrm{g} / \mathrm{mL}$ and $10 \mathrm{ng} / \mathrm{mL}$, respectively. The intensity of cyan indicates blocking strength ranging from $0 \%$ (no blocking, white) to 100\% (complete blocking, dark cyan). Red arrows indicate the representative nAbs (X01, X10 and X17). (F) Neutralization potencies of X01, X10 and X17 against SARS-CoV-2 VOCs, including B.1.1.7, B.1.1.28, B.1.351, B.1.617.2 and B.1.1.529. (G and H) Binding activities (G) 
840 and neutralization potencies $(H)$ of $X 01, X 10$ and $X 17$ against SARS-CoV-2 Omicron

841 variant. The $\mathrm{EC}_{50}$ and $\mathrm{IC}_{50}$ values were calculated by Prism software using with non842 linear regression (four parameters).

843 

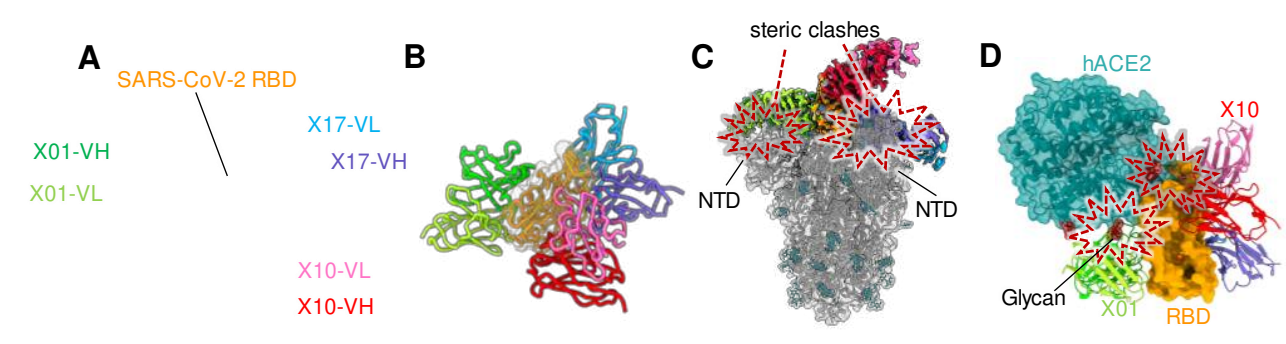

E

F $\quad$ G
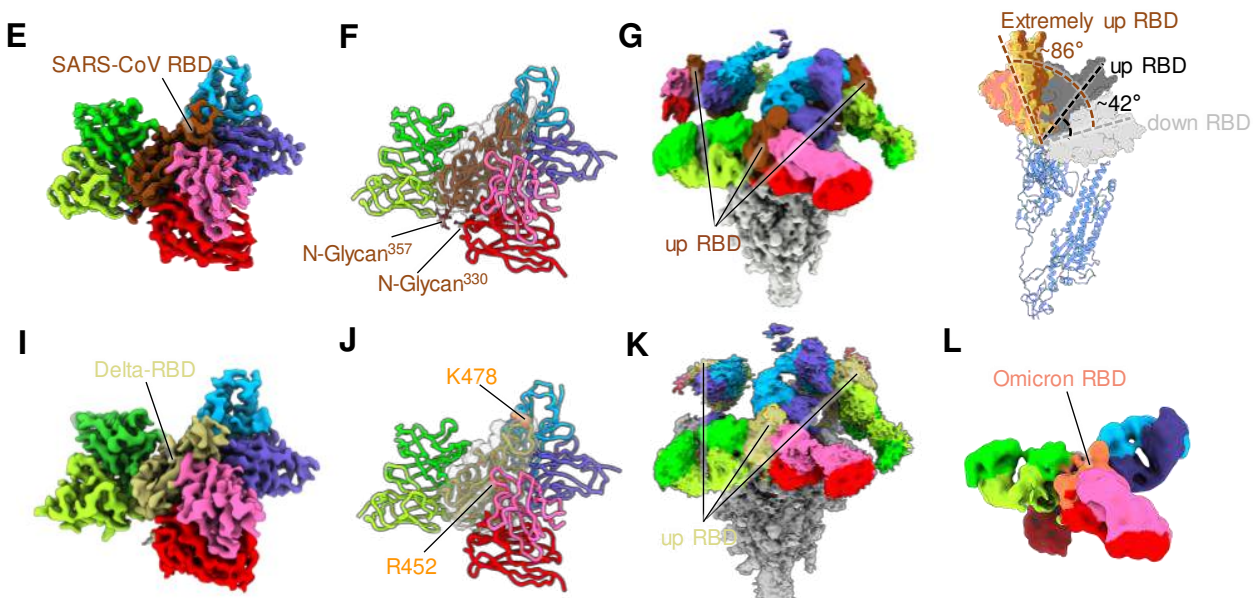

$\mathbf{J}$
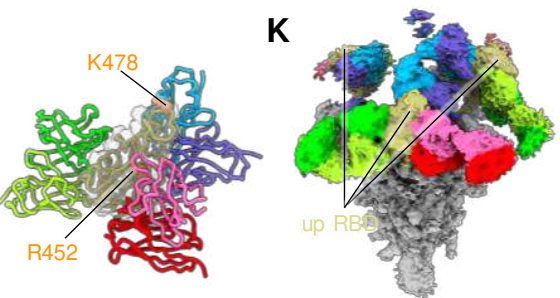

$\mathbf{L}$
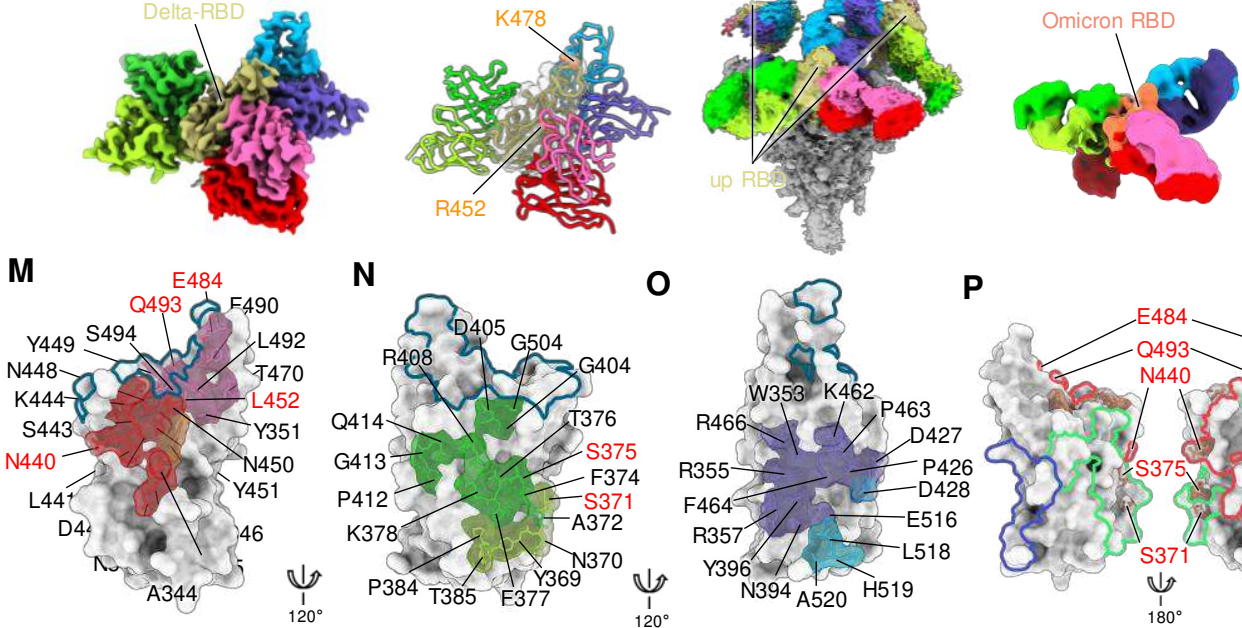

$\mathbf{N}$
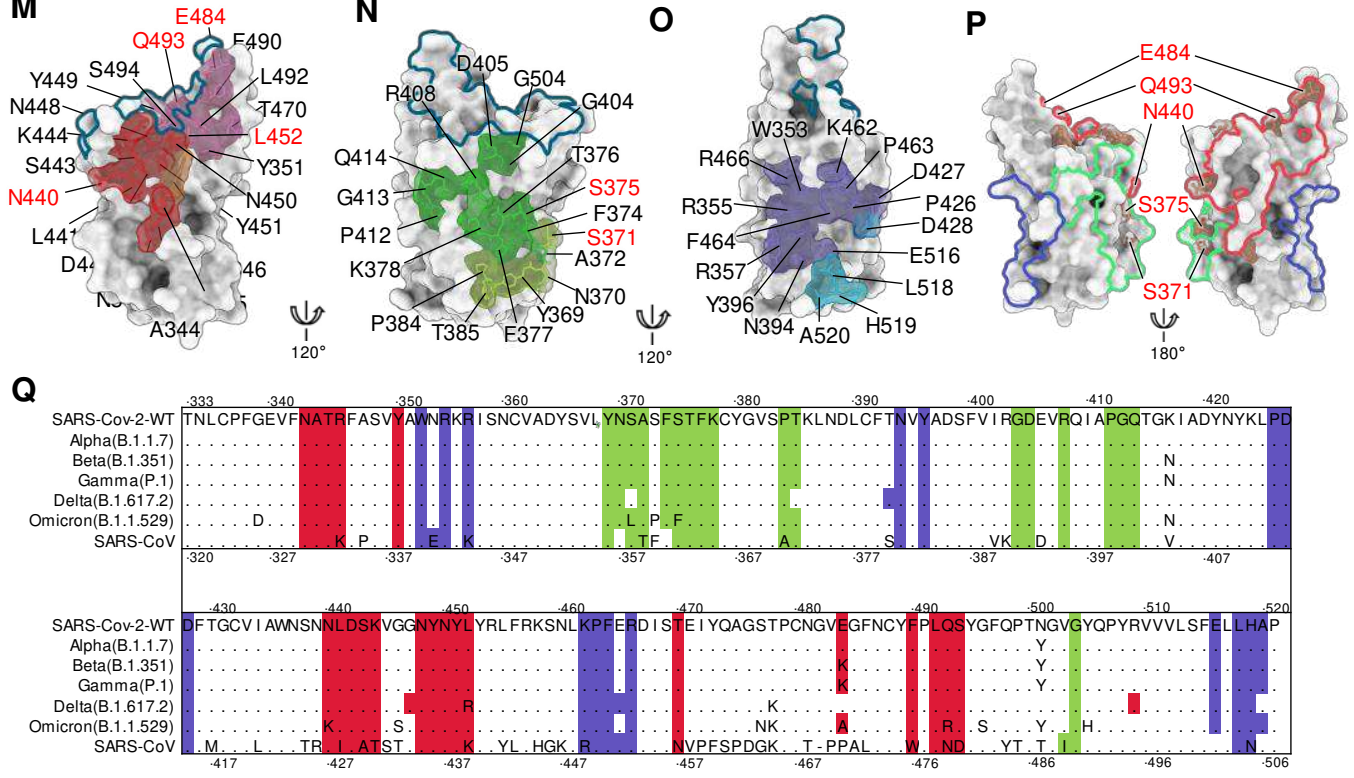

Fig. 2. Cryo-EM structures of three-antibody in complex with spike proteins of 
851

852

853

854

855

856

857

858

859

860

861

862

863

864

865

866

867

868

869

870

871

872

density map of SARS-CoV-2-S:X10:X01:X17 indicates the steric clashes (orange dashed boxes) between both Fab X01 and X17 and the neighboring NTD. (D) Superimposition of structures of ACE2:RBD (PDB: 6M0J) and SARS-CoV-2S:X10:X01:X17 shows the steric clashes between both Fab X01 and X10 and ACE2. (E-G) Cryo-EM structure of SARS-CoV-S:X10:X01:X17. Local refinement density map of interface (E), original global refinement density map (G) and the cartoon representation $(F)$ of the model were shown. $(\mathbf{H})$ The binding of three nAbs on trimeric spike caused the opening of all three RBDs and each RBD showed an extremely opened orientation of $\sim 90^{\circ} \quad$ (relative to closed RBD), compared to $\sim 42^{\circ}$ for the canonical up RBD. (I-K) Cryo-EM structure of Delta-S:X10:X01:X17. Local refinement density map of interface $(\mathrm{I})$, original global refinement density map $(\mathrm{K})$ and the cartoon representation $(\mathrm{J})$ of the model were shown. (L) The domain colored cryo-EM map of the cryo-EM structure of Omicron-S:X10:X01:X17. (M-O) Footprints of X10 (M), X01 (N) and X17 (O) on SARS-CoV-2 WT-RBD. The RBD was presented as surface representation (gray). The residues involved in nAbs interaction were shown as stick representation with transparent surface. The contact regions of the heavy, light chains and both chains of $\mathrm{X} 10$ on the RBD were colored in red, pink and brown, respectively. The contact regions of heavy and light chain of X01 were colored in lime and yellowgreen, respectively. The contact region of heavy and light chain of X17 were colored in slate blue and sky blue, respectively. The ACE2-binding site (base on PDB no. 7C8D) is marked as the black dotted-line. (P) No overlapping of footprints of X10 (red line), X01 (green line) and X17 (slate blue line) was observed on WT-RBD (gray surface 
873 representation). The mutation sites of Omicron variant on RBD are highlighted in coral.

874 (Q) Sequence alignment of the RBDs of SARS-CoV-2, VOCs and SARS-CoV with 875 strictly conserved residues shown as dots and the epitopes of three nAbs highlighted 876 with color scheme according to $(P)$.

877 

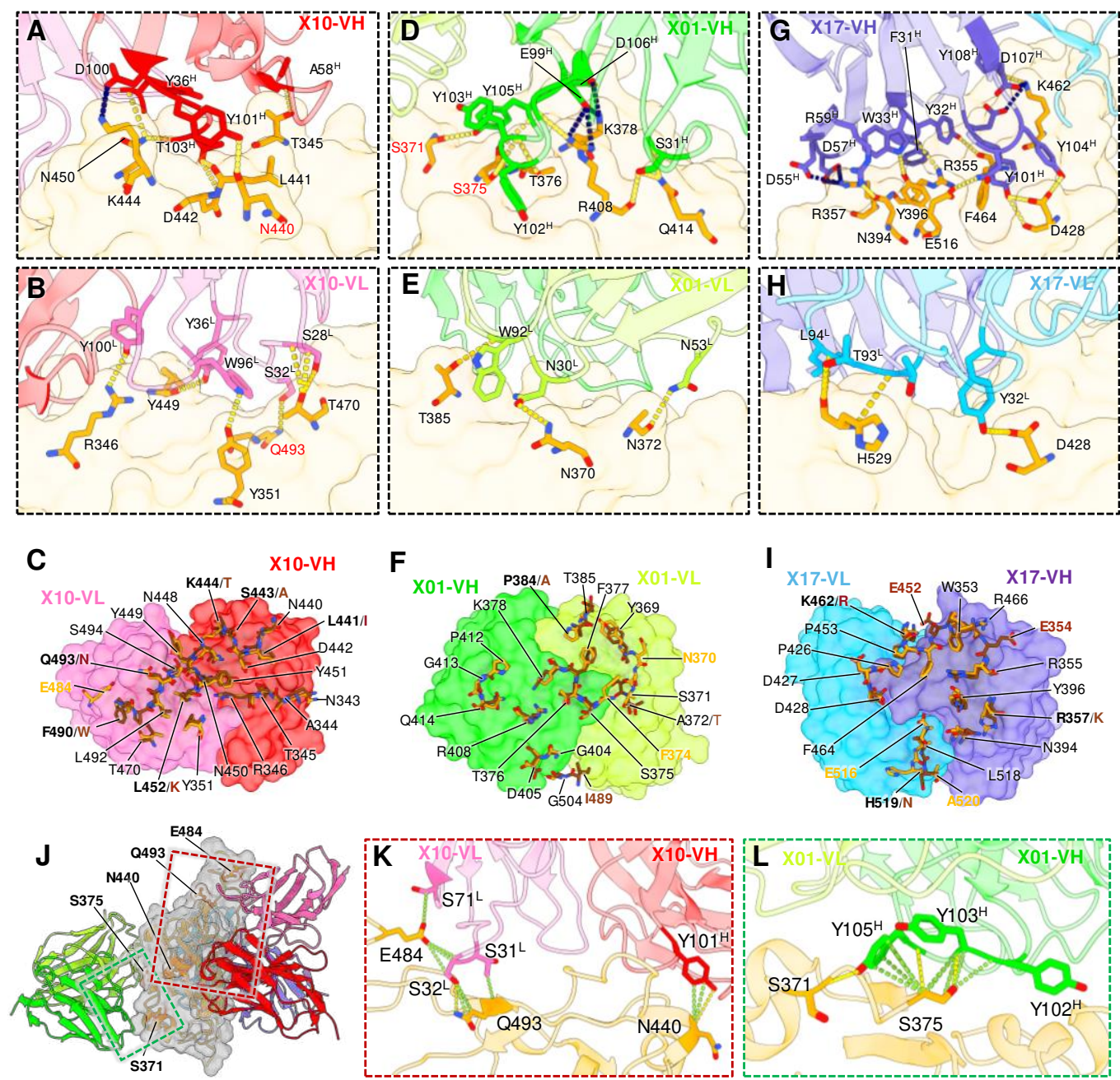

Fig. 3. Interactions details of three nAbs and the structural basis of decreased

neutralization of X10 and X01 against Omicron variant. (A and B) Interactions

mediated a network of hydrogen bonds (yellow dash lines) and salt bridges (dark blue of RBD was also shown, those residues that participated in hydrogen bonds or salt 
888

889

890

891

892

893

894

895

896

897

898

899

900

901

902

903 and E) Interactions details of SARS-CoV-2 WT-RBD bound by X01 heavy (D) and light chain (E). (F) The structural comparison of X01 epitopes on SARS-CoV-2 (orange stick) and SARS-CoV (brown stick). The VH (lime) and VL (yellow-green) of X01 were presented as a transparent surface. ( $\mathbf{G}$ and $\mathbf{H}$ ) Interactions details of SARS-CoV-2 WTRBD bound by X17 heavy (G) and light chain (H). (I) The structural comparison of X17 epitopes on SARS-CoV-2 (orange stick) and SARS-CoV (brown stick). The VH (slate blue) and VL (sky blue) of X17 were presented as a transparent surface. Residues on the RBDs involved in the interactions with three nAbs are labeled in black and those diverse residues on epitopes of SARS-CoV and SARS-CoV-2 were highlighted labeled (J) The structure of SARS-CoV-2-S:X10:X01:X17 with highlighted residues on WTRBD regarding to Omicron mutation (displayed in coral stick) that involved in nAbsRBD interaction. Three Fabs and RBD are shown as cartoon representation with transparent surface. ( $K$ and $\mathbf{L}$ ) Interaction details of $X 10(K)$ and $X 01(L)$ to those residues that are involved in Omicron mutations. Contacts and hydrogen bonds are marked as green and yellow dash lines, respectively. 


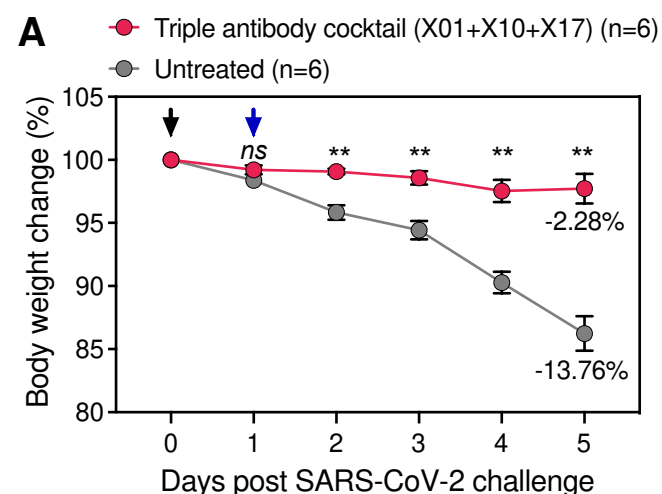

B

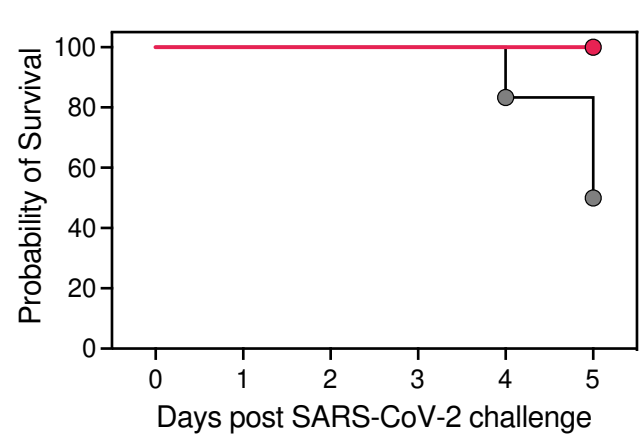

C

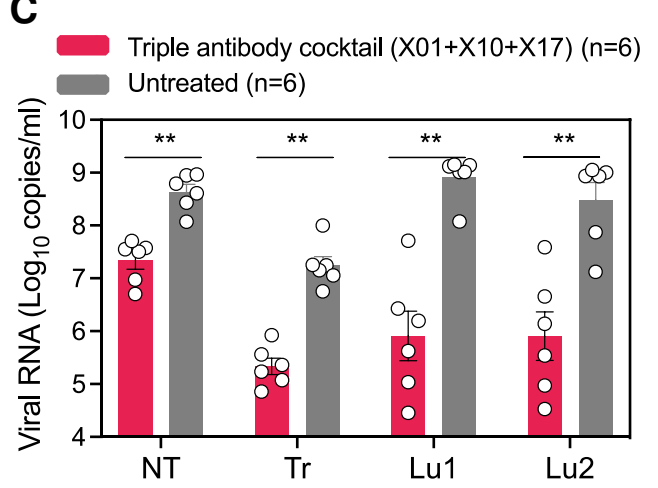

D Untreated group

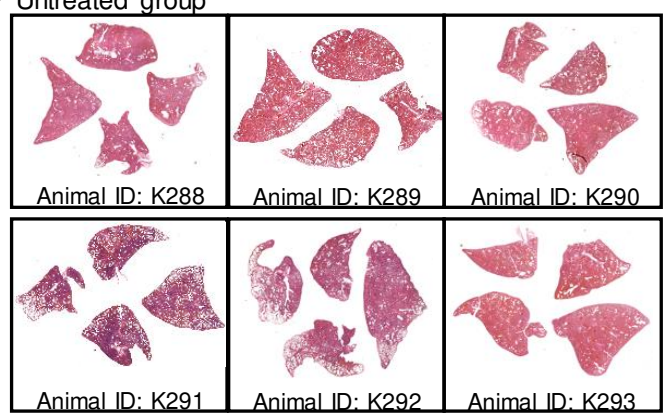

E Triple antibody cocktail group
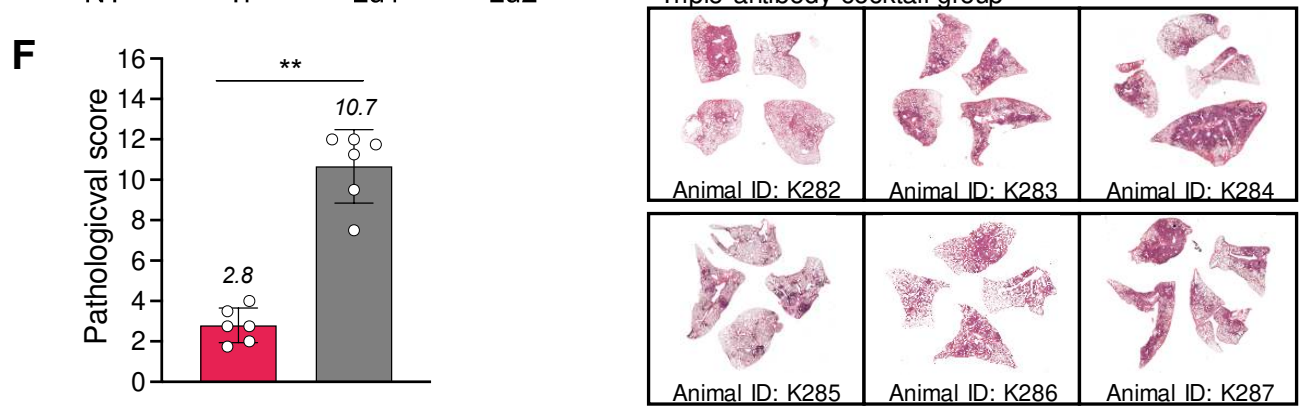

904

Fig. 4. Efficacy of triple antibody cocktail in protecting against SARS-CoV-2

B.1.351 infection in hamsters. (A) Groups of 6 hamsters were intravenously administered (blue arrow) by triple antibody cocktail (1:1:1 mixture of X01, X10 and $\mathrm{X} 17 \mathrm{nAbs}$ ) at a total dosage of $35 \mathrm{mg} / \mathrm{kg}$ (red) or PBS (gray) as control at one day post plotted. The average weight loss of each group at $5 \mathrm{dpi}$ is indicated. Data are means \pm SEM. $(B)$

(B) Kaplan-Meier survival plot.

(C) Concentrations of viral RNA in lysates of

912 the nasal turbinate (NT), trachea (Tr) and lung regions proximal (Lu1) and distal (Lu2) to the hilum from hamsters were quantified. Data are shown as means \pm SEM. The 
914 difference between the groups was analyzed by Mann Whitney test. (D and E) H\&E915 staining of four whole lung lobes collected from the PBS (untreated) group (D) and the 916 triple antibody cocktail treated group (E) at 5 dpi. (F) Pathological severity scores for 917 hamster lungs at $5 \mathrm{dpi}$. The average score of 4 independent lobes is calculated as the 918 pathological severity scores for individual hamsters. Data are shown as means \pm SEM. 919 The difference between the groups was analyzed by Mann Whitney test. Asterisks 920 indicate statistical significance (ns: not significant; ${ }^{* *}: \mathrm{P}<0.01$ ). 


\section{Supplementary Files}

This is a list of supplementary files associated with this preprint. Click to download.

- Supplementaryinformation.pdf

- ValidationreportSARSCoVSX10X01X17.pdf

- ValidationreportSARSCoV2SX10X01X17.pdf

- ValidationreportSARSCoVSX10X01X17interface.pdf

- ValidationreportDeltaSX10X01X17.pdf

- ValidationreportDeltaSX10X01X17interface.pdf

- ValidationreportOmicronSX10X01X17.pdf 